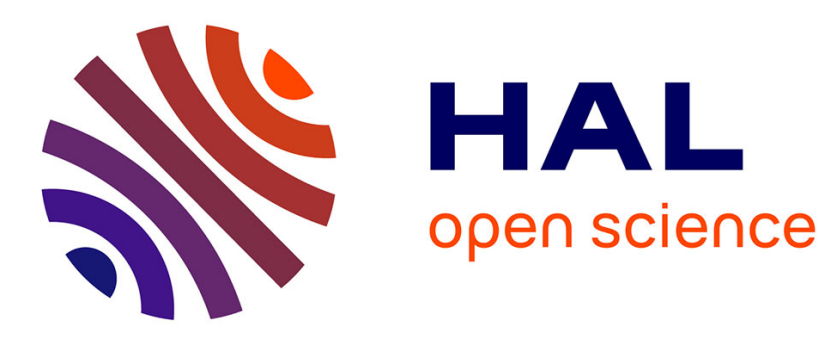

\title{
Stress gradient elasticity theory: existence and uniqueness of solution
}

Karam Sab, Frédéric Legoll, Samuel Forest

\section{To cite this version:}

Karam Sab, Frédéric Legoll, Samuel Forest. Stress gradient elasticity theory : existence and uniqueness of solution. Journal of Elasticity, 2016, 123 (2), pp.179-201. 10.1007/s10659-015-9554-1 . hal01288563

\section{HAL Id: hal-01288563 \\ https://hal.science/hal-01288563}

Submitted on 7 May 2019

HAL is a multi-disciplinary open access archive for the deposit and dissemination of scientific research documents, whether they are published or not. The documents may come from teaching and research institutions in France or abroad, or from public or private research centers.
L'archive ouverte pluridisciplinaire HAL, est destinée au dépôt et à la diffusion de documents scientifiques de niveau recherche, publiés ou non, émanant des établissements d'enseignement et de recherche français ou étrangers, des laboratoires publics ou privés. 


\title{
Stress Gradient elasticity theory: existence and uniqueness of solution
}

Karam Sab ${ }^{1}$, Frédéric Legoll ${ }^{1,2}$ and Samuel Forest ${ }^{3}$

${ }^{1}$ Université Paris-Est, Laboratoire Navier (ENPC, IFSTTAR, CNRS UMR 8205),

6 et 8 avenue Blaise Pascal, 77455 Marne-la-Vallée Cedex 2, France

${ }^{2}$ INRIA Rocquencourt, MATHERIALS project-team, 78153 Le Chesnay Cedex, France

${ }^{3}$ MINES ParisTech

Centre des matériaux, CNRS UMR 7633

BP 87, 91003 Evry Cedex, France

May 7, 2019

\begin{abstract}
The objective of the present article is to assess the well-posedness of the stress gradient linear elastic problems recently introduced by Forest and Sab (2012) and to formulate the corresponding existence and uniqueness theorems. In particular, we show that such theorems can be established in the case of the boundary value problems formulated in (Forest and Sab, 2012) with the corresponding boundary conditions.
\end{abstract}

Keywords Stress gradient elasticity. Higher order elasticity. Boundary conditions. Existence and uniqueness of solution.

Mathematics Subject Classification (2010) 35D30 . 35J56 . 35Q74.

\section{Introduction}

Strain gradient and stress gradient theories are two distinct continuum models for materials with fundamentally different kinematic, static and constitutive properties. The strain gradient elasticity initiated by Toupin (1962) and culminating in the works of Mindlin (1965); Mindlin and Eshel (1968) is based on the introduction of the second gradient of the displacement field (i.e., the strain gradient) in the free energy density of the material. The Aifantis gradient elasticity model, which has attracted much attention in the past twenty years and involves the Laplacian of the stress tensor in the constitutive equations, has been shown to be a special case of Mindlin's strain gradient elasticity (Ru and Aifantis, 1993; Forest and Aifantis, 2010). In contrast, the concept of stress gradient continuum came out only recently in the works of Forest and Sab (2012); Polizzotto

\footnotetext{
${ }^{1}$ Corresponding author. Tel.: +33-1-64-15-37-49; karam.sab@enpc.fr
} 
(2014, 2015), even though the notion of stress gradient was invoked in several earlier contributions without consistently building the specific balance and constitutive equations of that theory. The stress gradient model differs from the strain gradient theory by the fact that the dual quantity to the stress gradient involves new kinematic degrees of freedom, called microdisplacements by Forest and Sab (2012). This results in distinct boundary conditions and constitutive equations. The stress gradient model is hence not a particular case of strain gradient models, and we refer to (Forest and Sab, 2012, Table 1) for a comprehensive comparison of the two types of models. In turn, the formulations of the stress gradient theories presented by Forest and Sab (2012) and Polizzotto (2014) share several common features but differ in particular in the definition of essential and natural boundary conditions, i.e., generalized Dirichlet and Neumann boundary conditions. It can be shown however that both stress gradient theories lead, in a simplified isotropic case, to the same Eringen constitutive model involving the Laplacian of the stress tensor components (Eringen, 2002).

What are the proper boundary conditions for strain and stress gradient elasticity ensuring existence and uniqueness of solutions? This question is settled in the case of strain gradient elasticity thanks to the pioneering contributions by Mindlin (1965); Bleustein (1967); Mindlin and Eshel (1968); Germain (1973) and, more recently, Dell'Isola and Seppecher (1995, 1997); Dell'Isola et al. (2012); Iesan and Quintanilla (2013). Dirichlet boundary conditions amount to fix the displacement and the normal gradient of the displacement on the boundary of the domain, whereas Neumann boundary conditions involve a complex form of the first and second order traction vectors. Under appropriate conditions of definite-positiveness of the first and second order elastic moduli, existence and uniqueness of solutions are ensured.

The corresponding statements for stress gradient elasticity are not yet available in the literature and boundary conditions remain controversial: Forest and Sab (2012) claimed that free boundary conditions require that all the stress components be null on the boundary surface, whereas Polizzotto (2015) claimed that this is "very hard to satisfy" and suggested other conditions. The objective of the present article is to assess the well-posedness of stress gradient linear elastic problems and to formulate the corresponding existence and uniqueness theorems. In particular, we show here that such theorems can be established in the case of the boundary value problems formulated in (Forest and Sab, 2012) and Polizzotto (2015) with the corresponding boundary conditions. We consider here three types of boundary conditions: (i) clamping boundary conditions (i.e., Dirichlet conditions, which amount to prescribing the generalized displacement) in Sections 3, 4 and 5, (ii) free boundary conditions (i.e., Neumann conditions, which amount to prescribing the generalized stress like in (Forest and Sab, 2012)) in Section 6, and (iii) mixed boundary conditions ( which amount to prescribing some components of the generalized stress and some components of the generalized displacement like in Polizzotto (2015)) in Section 7.

Existence and uniqueness theorems for generalized continua remain seldom in the literature. Some of them have been established for the linear elastic Cosserat continuum (Iesan, 2007; Jeong and Neff, 2010) and for Eringen's micromorphic continuum (Iesan and Quintanilla, 1994; Neff and Forest, 2007). There is a current debate on the choice of suitable boundary conditions for stress gradient media, as discussed in Forest and Sab (2012) 
and Polizzotto (2014, 2015). In the original work (Forest and Sab, 2012), the static boundary conditions amount to fixing all stress tensor components on the boundary of the domain, whereas only the usual traction vector is prescribed in the theory by Polizzotto (2014). There is therefore a need for mathematical statements on existence and uniqueness of solutions in linear elasticity to assess the proposed boundary conditions. In the present work, we prove such theorems in the case of stress gradient elasticity for the boundary conditions stated in (Forest and Sab, 2012).

Our article is organized as follows. After setting the notations used throughout this work in Section 2, the balance and constitutive equations of the stress gradient theory are recalled in the context of linear anisotropic elasticity in Section 3. We introduce there a stress formulation based on the complementary energy, and show its well-posedness (see Theorem 3). In Section 4, we turn to a displacement formulation based on minimizing the potential energy. We assume there clamping boundary conditions (in the spirit of Dirichlet boundary conditions on the displacement). The main technical difficulty is to show the coercivity of the stress gradient elasticity potential, which is the aim of Section 4.2. Using this result, the existence and uniqueness of the solution is established in Section 4.3 (see Theorem 6). We next study (see Section 5) the relation between the stress formulation introduced in Section 3 and the displacement formulation introduced in Section 4. In Section 6, we eventually consider free boundary conditions, in the spirit of Neumann boundary conditions, and establish the well-posedness of the corresponding formulation (see Theorem 10). We point out several possible generalizations of our approach in Section 7, and collect concluding remarks in Section 8.

\section{Notations and preliminaries}

We collect here some notations concerning tensorial calculus and functional spaces, and recall some links between the symmetrized gradient of a displacement field and rigid body displacements.

\subsection{Tensorial calculus}

All the vector spaces considered in this article are over $\mathbb{R}$, and the space dimension is 3. Throughout this article, Latin indices vary in the set $\{1,2,3\}$ when they are not used for indexing sequences, and the summation convention with respect to repeated indices is systematically used in conjunction with this rule. Tensors of zeroth, first, second, third, fourth, fifth and sixth ranks are respectively denoted by $a, \underline{\boldsymbol{a}}, \underset{\sim}{\boldsymbol{a}}, \underset{\boldsymbol{a}}{\underset{\sim}{\boldsymbol{a}}} \underset{\sim}{\boldsymbol{a}}$ and $\underset{\sim}{\boldsymbol{a}}$. To avoid any confusion, the intrinsic notation is usually complemented by the index notation with reference to a Cartesian orthonormal basis $\left(\underline{\boldsymbol{e}}_{1}, \underline{\boldsymbol{e}}_{2}, \underline{\boldsymbol{e}}_{3}\right)$. The space of first, second and third rank tensors are respectively denoted by $\underline{\mathbb{R}}$ (so that $\underline{\mathbb{R}}=\mathbb{R}^{3}$ ), $\underset{\sim}{\mathbb{R}}$ and $\mathbb{R}$. The subscript $S$ appended to $\underset{\sim}{\mathbb{R}}$ and $\mathbb{R}$ denotes the space of symmetric second and third rank tensors with respect to the first two indices:

$$
\forall \underset{\sim}{\boldsymbol{a}}=\left(a_{i j}\right) \in \mathbb{\sim}_{S}^{\mathbb{R}_{S}}, \quad a_{i j}=a_{j i} \quad \text { and } \quad \forall \underline{\boldsymbol{a}}=\left(a_{i j k}\right) \in \mathbb{R}_{S}, \quad a_{i j k}=a_{j i k} .
$$


The dimension of the vector space $\underset{\sim}{\mathbb{R}}$ (resp. $\mathbb{R}_{S}$ ) is therefore 6 (resp. 18). These spaces are respectively endowed with the following scalar products:

$$
\underset{\sim}{\boldsymbol{a}}: \underset{\sim}{\boldsymbol{a}^{\prime}}=a_{i j} a_{i j}^{\prime}, \quad \underline{\boldsymbol{a}} \therefore \underline{\boldsymbol{a}}^{\prime}=a_{i j k} a_{i j k}^{\prime}
$$

For any $\underline{\boldsymbol{a}} \in \mathbb{R}_{S}$ and any $\underset{\sim}{\boldsymbol{b}} \in \mathbb{\sim}_{S}$, we define

$$
\underline{\boldsymbol{a}}: \underset{\boldsymbol{b}}{=}=a_{i j k} b_{j k} \underline{\boldsymbol{e}}_{i} .
$$

We recall that any symmetric second rank tensor $\underset{\sim}{\boldsymbol{a}} \in \mathbb{R}_{\sim}$ can be decomposed into a spherical part $\underset{\sim}{\boldsymbol{a}} \in \mathbb{\sim}_{S}^{s} \subset \underset{\sim}{\mathbb{R}_{S}}$ and a deviatoric part $\underset{\sim}{\boldsymbol{a}^{\tilde{d}}} \in \mathbb{\sim}_{\sim}^{\tilde{R}^{d}} \subset \mathbb{\sim}_{S}$ as

$$
\underset{\sim}{\boldsymbol{a}}={\underset{\sim}{s}}^{s}+{\underset{\sim}{\boldsymbol{a}}}^{d}
$$

with

$$
{\underset{\sim}{\boldsymbol{a}}}^{s}=\frac{1}{3}(\operatorname{Tr} \underset{\sim}{\boldsymbol{a}}) \underset{\sim}{\mathbf{1}}
$$

where $\underset{\sim}{\mathbf{1}}$ is the second rank identity tensor. We see that

$$
{\underset{\sim}{\boldsymbol{a}}}^{s}:{\underset{\sim}{\boldsymbol{a}}}^{d}=0
$$

The space of spherical second rank tensors is $\mathbb{R}_{S}^{s}=\{a \underset{\sim}{\mathbf{1}}, a \in \mathbb{R}\}$ while the space of deviatoric second rank tensors is $\underset{\sim}{\mathbb{R}} d=\left\{\underset{\sim}{\boldsymbol{a}} \in \mathbb{\sim}_{S}, \operatorname{Tr} \underset{\sim}{\boldsymbol{a}}=0\right\}$.

A similar decomposition can be introduced for third rank tensors. Let

$$
\underline{\mathbb{R}}_{S}^{d}=\left\{\underline{\boldsymbol{a}} \in{\underset{\mathbb{R}}{S}}_{S}, \underline{\boldsymbol{a}}: \underset{\sim}{\mathbf{1}}=0\right\}=\left\{\underline{\boldsymbol{a}} \in \underline{\mathbb{R}}_{S}, a_{i j k} \delta_{j k}=0\right\}
$$

be the space of deviatoric symmetric third rank tensors, where $\delta_{j k}$ is the Kronecker symbol. Let $\underline{\mathbb{R}}_{S}^{s}=\left(\underline{\mathbb{R}}_{S}^{d}\right)^{\perp}$, so that $\underline{\mathbb{R}}_{S}=\underline{\mathbb{R}}_{S}^{s} \oplus \underline{\mathbb{R}}_{S}^{d}$. Any symmetric third rank tensor $\underline{\boldsymbol{a}} \in \underline{\mathbb{R}}_{S}$ can be decomposed into a spherical part $\underline{\boldsymbol{a}}^{s} \in \mathbb{R}_{S}^{s} \subset \mathbb{R}_{S}$ and a deviatoric part $\underline{\boldsymbol{a}}^{d} \in \mathbb{R}_{S}^{d} \subset \mathbb{R}_{S}$ as

$$
\underline{\boldsymbol{a}}=\underline{\boldsymbol{a}}^{s}+\underline{\boldsymbol{a}}^{d}
$$

with

$$
a_{i j k}^{S}=\frac{1}{4}\left(a_{i l m} \delta_{l m} \delta_{j k}+a_{j l m} \delta_{l m} \delta_{i k}\right) .
$$

Remark 1. In the two-dimensional case, the formula (1) should be replaced by

$$
a_{i j k}^{s}=\frac{1}{3}\left(a_{i l m} \delta_{l m} \delta_{j k}+a_{j l m} \delta_{l m} \delta_{i k}\right),
$$

where the indices $i, j$ and $k$ only take the value 1 or 2.

The tensor product is denoted by $\otimes$. We define the symmetrized tensor product as

$$
\underline{\boldsymbol{a}} \stackrel{\stackrel{s}{\otimes}}{\boldsymbol{b}}=\frac{1}{2}(\underline{\boldsymbol{a}} \otimes \underline{\boldsymbol{b}}+\underline{\boldsymbol{b}} \otimes \underline{\boldsymbol{a}}), \quad a_{(i} b_{j)}=\frac{1}{2}\left(a_{i} b_{j}+a_{j} b_{i}\right) .
$$


The nabla operator is denoted by $\underline{\boldsymbol{\nabla}}$, with $\boldsymbol{\nabla}_{i}=\frac{\partial}{\partial x_{i}}$. It operates as follows on a vector field $\underline{\boldsymbol{u}}$ :

$$
\underline{\boldsymbol{u}} \otimes \underline{\boldsymbol{\nabla}}=\frac{\partial u_{i}}{\partial x_{j}} \underline{\boldsymbol{e}}_{i} \otimes \underline{\boldsymbol{e}}_{j}=u_{i, j} \underline{\boldsymbol{e}}_{i} \otimes \underline{\boldsymbol{e}}_{j}
$$

The Cauchy stress tensor is a symmetric second rank tensor denoted by $\boldsymbol{\sigma}$, which has the following components:

$$
\underset{\sim}{\boldsymbol{\sigma}}=\sigma_{i j} \underline{\boldsymbol{e}}_{i} \otimes \underline{\boldsymbol{e}}_{j}
$$

Its divergence is the vector

$$
\underline{\boldsymbol{\sigma}} \cdot \underline{\boldsymbol{\nabla}}=\sigma_{i j, j} \underline{\boldsymbol{e}}_{i}
$$

The stress gradient tensor is a third rank tensor, defined by

$$
\underline{\boldsymbol{\sigma}} \otimes \underline{\boldsymbol{\nabla}}=\sigma_{i j, k} \underline{\boldsymbol{e}}_{i} \otimes \underline{\boldsymbol{e}}_{j} \otimes \underline{\boldsymbol{e}}_{k}
$$

Its components are symmetric with respect to the first two indices. It is hence in $\mathbb{R}_{S}$. We note that the spherical part of the stress gradient is directly related to the divergence of the stress tensor by

$$
(\underset{\sim}{\boldsymbol{\sigma}} \otimes \underline{\boldsymbol{\nabla}})_{i j k}^{s}=\frac{1}{4}\left(\sigma_{i m, m} \delta_{j k}+\sigma_{j m, m} \delta_{i k}\right)=\frac{1}{4}\left(\left[(\underset{\boldsymbol{\sigma}}{\boldsymbol{\nabla}} \underline{\boldsymbol{\nabla}}) \cdot \underline{\boldsymbol{e}}_{i}\right] \delta_{j k}+\left[(\boldsymbol{\boldsymbol { \sigma }} \cdot \underline{\boldsymbol{\nabla}}) \cdot \underline{\boldsymbol{e}}_{j}\right] \delta_{i k}\right)
$$

or equivalently,

$$
(\boldsymbol{\sigma} \otimes \underline{\boldsymbol{\nabla}}): \underset{\sim}{\mathbf{1}}=(\underset{\sim}{\boldsymbol{\sigma}} \otimes \underline{\boldsymbol{\nabla}})^{s}: \underset{\sim}{\mathbf{1}}=\boldsymbol{\sigma} \cdot \underline{\boldsymbol{\nabla}} .
$$

In particular

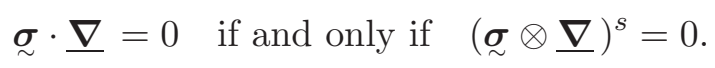

\subsection{Functional spaces}

We consider a bounded, connected, open subset $\Omega$ of $\mathbb{R}^{3}$ whose boundary is Lipschitzcontinuous in the sense of Necas (1967) or Adams (1975). Usual notations of functional analysis are used. So, $D(\Omega)$ denotes the space of functions defined on $\Omega$, that are infinitely differentiable and have compact support. In turn, $D^{\prime}(\Omega)$ denotes the space of distributions defined over $\Omega$. The notations $H^{m}(\Omega), m \in \mathbb{Z}$, with $H^{0}(\Omega)=L^{2}(\Omega)$, and $H_{0}^{1}(\Omega)$ denote the usual Sobolev spaces. Spaces of vector fields and tensor fields are denoted according to the tensorial notations introduced in Section 2.1. The subscript $S$ appended to a special Roman capital letter denotes a space of symmetric tensor fields. For instance, $\underline{\boldsymbol{D}}(\Omega)$ denotes the space of vector fields having their components in $D(\Omega), \underset{\sim}{\boldsymbol{D}}(\Omega)$ denotes the space of symmetric second rank tensor fields having their components in $D(\Omega), D_{S}(\Omega)$ denotes the space of third rank tensor fields being symmetric with respect to the first two indices and having their components in $D(\Omega)$. Likewise, $\underline{\boldsymbol{L}}^{2}(\Omega), \underset{\sim}{\boldsymbol{L}} \mathbf{S}(\Omega)$ and $\underline{\boldsymbol{L}}_{S}^{2}(\Omega)$ denote, respectively, the space of vector fields, symmetric second rank fields and symmetric third rank fields having their components in $L^{2}(\Omega)$. The $L^{2}$ norm involves the natural scalar product on these spaces. Hence, it is defined by

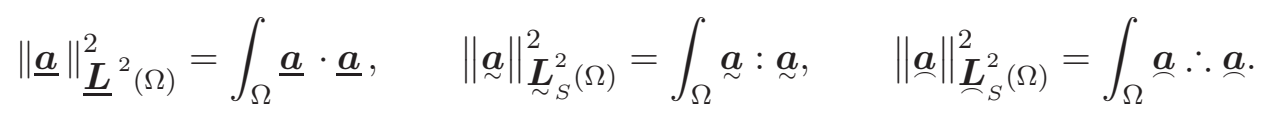

The following theorems will be useful in the sequel. Let $\underline{\boldsymbol{u}} \in \underline{\boldsymbol{D}}^{\prime}(\Omega)$ : 
- The distribution $\underline{\boldsymbol{u}} \stackrel{s}{\otimes} \underline{\boldsymbol{\nabla}} \in \underline{\sim}_{S}^{\prime}(\Omega)$ vanishes if, and only if, there exist some constants $\underline{\boldsymbol{t}} \in \underline{\mathbb{R}}$ and $\underline{\boldsymbol{\omega}} \in \underline{\mathbb{R}}$ such that $\underline{\boldsymbol{u}}$ is a function satisfying (Moreau, 1979)

$$
\forall \underline{x} \in \Omega, \quad \underline{\boldsymbol{u}}(\underline{\boldsymbol{x}})=\underline{\boldsymbol{t}}+\underline{\boldsymbol{\omega}} \times \underline{\boldsymbol{x}},
$$

where $\underline{\boldsymbol{t}}$ and $\underline{\boldsymbol{\omega}}$ are respectively called the translation vector and the rotation vector of the infinitesimal rigid body displacement field $\underline{\boldsymbol{u}}$. We denote by $\underline{\mathcal{R}}$ the vector space of such rigid body displacement fields:

$$
\underline{\mathcal{R}}=\{\underline{\boldsymbol{u}} \text { is a function of the form (5) }\} .
$$

- The distribution $\underline{\boldsymbol{u}} \stackrel{s}{\otimes} \underline{\boldsymbol{\nabla}}$ is in ${\underset{\sim}{\boldsymbol{H}}}_{S}^{-1}(\Omega)$ if, and only if, $\underline{\boldsymbol{u}}$ is in $\underline{\boldsymbol{L}}^{2}(\Omega)$ (see (Amrouche et al., 2006, Theorem 3.1)). Moreover, in view of (Amrouche et al., 2006, Proof of Theorem 3.2), there exists a constant $C$ such that

$$
\forall \underline{\boldsymbol{u}} \in \underline{\boldsymbol{L}}^{2}(\Omega), \quad \inf _{\underline{\boldsymbol{r}} \in \underline{\mathcal{R}}}\|\underline{\boldsymbol{u}}-\underline{\boldsymbol{r}}\|_{\underline{\boldsymbol{L}}^{2}(\Omega)} \leq C\|\underline{\boldsymbol{u}} \stackrel{s}{\otimes} \underline{\boldsymbol{\nabla}}\|_{\boldsymbol{H}_{S}^{-1}(\Omega)} .
$$

\section{Formulation of the stress gradient elasticity model according to (Forest and Sab, 2012)}

We consider a homogeneous elastic stress gradient material occupying the domain $\Omega$. Its complementary energy is given by

$$
\mathcal{P}^{*}(\underset{\sim}{\boldsymbol{\sigma}})=\int_{\Omega} w^{*}\left(\underset{\sim}{\boldsymbol{\sigma}},(\underset{\sim}{\boldsymbol{\sigma}} \otimes \underline{\boldsymbol{\nabla}})^{d}\right),
$$

where we introduced the stress energy density potential $w^{*}(\underset{\sim}{\boldsymbol{\sigma}}, \underline{\boldsymbol{R}})$, which is assumed to be a symmetric definite positive quadratic form of the variables $\underset{\sim}{\boldsymbol{\sigma}} \in \mathbb{\sim}_{S}$ and $\boldsymbol{R} \in \mathbb{R}_{S}^{d}$. In what follows, we assume that $w^{*}$ does not couple $\boldsymbol{\sigma}$ and $\underline{\boldsymbol{R}}$ (this for instance holds in the case of point symmetry). There thus exist a fourth order stress compliance tensor $\underset{\approx}{\boldsymbol{S}^{\sigma}}$ (with $S_{i j k l}^{\sigma}=S_{j i k l}^{\sigma}=S_{k l i j}^{\sigma}$ ) and a sixth order stress gradient compliance tensor ${\underset{\boldsymbol{S}}{ }}^{R}$ (with $\left.S_{i j k l m n}^{R}=S_{j i k l m n}^{R}=S_{l m n i j k}^{R}\right)$ such that

$$
w^{*}(\underset{\sim}{\boldsymbol{\sigma}}, \underline{\boldsymbol{R}})=\frac{1}{2} \boldsymbol{\sigma}:{\underset{\sim}{\boldsymbol{S}}}^{\sigma}: \underset{\sim}{\boldsymbol{\sigma}}+\frac{1}{2} \underline{\boldsymbol{R}} \therefore \stackrel{\boldsymbol{S}}{R}^{R} \underset{\boldsymbol{R}}{ },
$$

where $\underset{\approx}{\boldsymbol{S}^{\sigma}}$ and ${\underset{\boldsymbol{S}}{R}}^{R}$ are positive definite in the sense that there exists $c>0$ such that

$$
\begin{aligned}
& \forall \underset{\sim}{\boldsymbol{\sigma}} \in{\underset{\sim}{\mathbb{R}}}_{S}, \quad \underset{\sim}{\boldsymbol{\sigma}}:{\underset{\sim}{\boldsymbol{S}}}^{\sigma}: \underset{\sim}{\boldsymbol{\sigma}} \geq c \boldsymbol{\sim}: \underset{\sim}{\boldsymbol{\sigma}}, \\
& \forall \underline{\boldsymbol{R}} \in \underline{\mathbb{R}}_{S}^{d}, \quad \underline{\boldsymbol{R}} \therefore \underline{S}^{R} \therefore \underline{\boldsymbol{R}} \geq c \underline{\boldsymbol{R}} \therefore \underline{\boldsymbol{R}} .
\end{aligned}
$$

We hence see that $w^{*}(\underset{\sim}{\boldsymbol{g}}, \underline{\boldsymbol{R}})$ vanishes only when $(\underset{\sim}{\boldsymbol{\sigma}}, \underline{\boldsymbol{R}})=(\underset{\sim}{\mathbf{0}}, \underline{\mathbf{0}})$. 
Remark 2. To keep notations simple, we assume here that the material is homogeneous, which implies that ${\underset{\sim}{\boldsymbol{S}}}^{\sigma}$ and $\underline{\boldsymbol{S}}^{R}$ do not depend on the spatial variable $\underline{\boldsymbol{x}}$. Our approach carries over to the case when $\underset{\approx}{\boldsymbol{S}}$ and ${\underset{\boldsymbol{S}}{\boldsymbol{S}}}^{R}$ depend on $\underline{\boldsymbol{x}}$. The assumption (10) should then be replaced by: $\underset{\approx}{\boldsymbol{S}} \in \underset{\approx}{\boldsymbol{L}} \boldsymbol{\infty}(\Omega)$ and there exists c $>0$ such that

$$
\forall \underset{\sim}{\boldsymbol{\sigma}} \in \mathbb{\sim}_{\sim}, \quad \underset{\sim}{\boldsymbol{\sigma}}:{\underset{\sim}{\boldsymbol{S}}}^{\sigma}(\underline{\boldsymbol{x}}): \underset{\sim}{\boldsymbol{\sigma}} \geq c \boldsymbol{\sigma}: \underset{\sim}{\boldsymbol{\sigma}} \quad \text { almost everywhere on } \Omega
$$

and likewise for (11).

The solid is subjected to body forces $\underline{\boldsymbol{f}} \in \underline{\boldsymbol{L}}^{2}(\Omega)$ and clamping conditions are imposed at its boundary $\partial \Omega$. Introduce the space of statically admissible generalized stress fields

$$
S A(\underline{\boldsymbol{f}})=\left\{\underset{\sim}{\boldsymbol{\sigma}} \in{\underset{\sim}{\boldsymbol{H}}}_{S}^{1}(\Omega), \quad \underline{\boldsymbol{\sigma}} \cdot \underline{\boldsymbol{\nabla}}+\underline{\boldsymbol{f}}=0 \text { on } \Omega\right\} .
$$

The variational formulation of the problem consists in minimizing the complementary energy with respect to all statically admissible generalized stress fields:

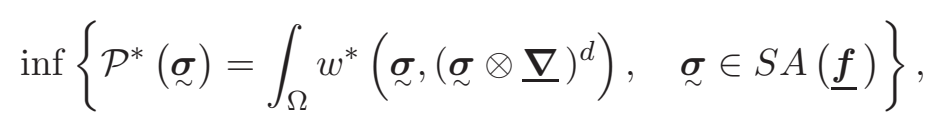

where $w^{*}$ is given by (9). Note that, when $\underset{\sim}{\boldsymbol{\sigma}} \in S A(\underline{\boldsymbol{f}})$, the divergence of $\underset{\sim}{\boldsymbol{\sigma}}$ and therefore, in view of (2), the spherical part of the stress gradient, is entirely determined. It is hence natural that only the deviatoric part of the stress gradient enters the stress energy density potential $w^{*}$.

\subsection{Well-posedness of the stress formulation (13)}

We have the following result:

Theorem 3. Under assumptions (10) and (11), the problem (9)-(13) has a unique solution $\boldsymbol{\sigma}^{\#}$. This field is also the unique solution in $S A(\underline{f})$ of the following variational formulation: find $\boldsymbol{\sigma}^{\#} \in S A(\underline{\boldsymbol{f}})$ such that

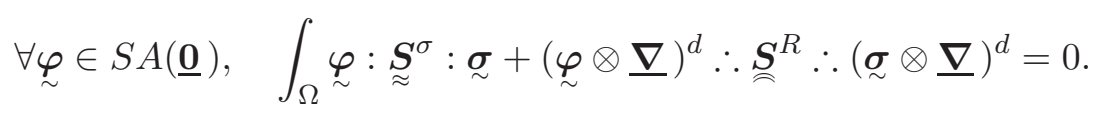

Proof. The proof is straightforward. We provide it for the sake of completeness. Introduce the symmetric bilinear form

$$
a_{s}(\underset{\sim}{\boldsymbol{\varphi}}, \boldsymbol{\sigma})=\int_{\Omega} \underset{\sim}{\boldsymbol{\varphi}}:{\underset{\sim}{\boldsymbol{S}}}^{\sigma}: \underset{\sim}{\boldsymbol{\sigma}}+(\underset{\sim}{\boldsymbol{\varphi}} \otimes \underline{\boldsymbol{\nabla}})^{d} \therefore \underline{\sim}^{R} \therefore(\underset{\sim}{\boldsymbol{\sigma}} \otimes \underline{\boldsymbol{\nabla}})^{d}
$$

so that $\mathcal{P}^{*}(\underset{\sim}{\boldsymbol{\sigma}})=\frac{1}{2} a_{s}(\underset{\sim}{\boldsymbol{\sigma}}, \boldsymbol{\sigma})$. Let ${\underset{\sim}{\boldsymbol{\sigma}}}^{f}$ be any element in $S A(\underline{\boldsymbol{f}})$. Then the problem (13) is equivalent to

$$
\inf \left\{\frac{1}{2} a_{s}(\underset{\sim}{\boldsymbol{\sigma}}, \underset{\sim}{\boldsymbol{\sigma}})+a_{s}\left({\underset{\boldsymbol{\sigma}}{f}}^{f}, \boldsymbol{\sigma}\right), \quad \underset{\sim}{\boldsymbol{\sigma}} \in S A(\underline{\mathbf{0}})\right\}
$$


The space $S A(\underline{\mathbf{0}})$, endowed with the scalar product

$$
\langle\underset{\sim}{\boldsymbol{\varphi}}, \underset{\sim}{\boldsymbol{\sigma}}\rangle_{S A}=\int_{\Omega} \underset{\sim}{\boldsymbol{\varphi}}: \underset{\sim}{\boldsymbol{\sigma}}+(\underset{\sim}{\boldsymbol{\varphi}} \otimes \underline{\boldsymbol{\nabla}}) \therefore(\underset{\sim}{\boldsymbol{\sigma}} \otimes \underline{\boldsymbol{\nabla}}),
$$

is a Hilbert space. The bilinear form $a_{s}$ is continuous on $S A(\underline{\mathbf{0}})$. In view of (10)-(11) and (4), it is also coercive on $S A(\underline{\mathbf{0}})$ :

$$
a_{s}(\underset{\sim}{\boldsymbol{\sigma}}, \underset{\sim}{\boldsymbol{\sigma}}) \geq c \int_{\Omega} \underset{\sim}{\boldsymbol{\sigma}}: \underset{\sim}{\boldsymbol{\sigma}}+(\underset{\sim}{\boldsymbol{\sigma}} \otimes \underline{\boldsymbol{\nabla}})^{d} \therefore(\underset{\sim}{\boldsymbol{\sigma}} \otimes \underline{\boldsymbol{\nabla}})^{d}=c\langle\underset{\sim}{\boldsymbol{\sigma}}, \underset{\sim}{\boldsymbol{\sigma}}\rangle_{S A}
$$

Using the Lax-Milgram theorem on (15), we deduce that the problem (13) is well-posed. Its unique solution $\boldsymbol{\sigma}^{\#} \in S A(\underline{\boldsymbol{f}})$ satisfies

$$
\forall \varphi \in S A(\underline{\mathbf{0}}), \quad a_{s}\left(\underset{\sim}{\boldsymbol{\varphi}}, \underline{\sim}^{\#}\right)=0,
$$

which is exactly (14).

\subsection{Displacement formulation}

The direct formulation of (13), i.e., a formulation in terms of generalized displacement fields, has been obtained by Forest and Sab (2012) using standard variational techniques. Let us introduce the strain fields $\underset{\sim}{e}$ and $\Phi$, which are conjugate through the stress energy density $w^{*}$ to the stress fields $\underset{\sim}{\boldsymbol{\sigma}}$ and $\underline{\boldsymbol{R}}=(\underset{\sim}{\boldsymbol{\sigma}} \otimes \underline{\boldsymbol{\nabla}})^{d}$ :

$$
\underset{\sim}{\boldsymbol{e}}=\frac{\partial w^{*}}{\partial \underset{\sim}{\boldsymbol{\sigma}}}(\underset{\sim}{\boldsymbol{\sigma}}, \underline{\boldsymbol{R}})={\underset{\sim}{\boldsymbol{S}}}^{\sigma}: \underset{\sim}{\boldsymbol{\sigma}}, \quad \underline{\Phi}=\frac{\partial w^{*}}{\partial \underline{\boldsymbol{R}}}(\underset{\sim}{\boldsymbol{\sigma}}, \underline{\boldsymbol{R}})=\underline{\sim}^{R} \therefore \underline{\boldsymbol{R}} .
$$

Note that the new kinematic degrees of freedom $\Phi$ form a deviatoric third order tensor. They are called microdisplacements in (Forest and Sab, 2012). The above relations play the role of constitutive equations in the model.

It was found that the field $\underset{\sim}{e}$ can be written in terms of $\boldsymbol{\Phi}$ and of a displacement vector field $\underline{\boldsymbol{u}}$ as

$$
\underset{\sim}{\boldsymbol{e}}=\underline{\boldsymbol{u}} \stackrel{s}{\otimes} \underline{\boldsymbol{\nabla}}+\underline{\boldsymbol{\Phi}} \cdot \underline{\boldsymbol{\nabla}}, \quad \text { i.e., } \quad e_{i j}=\frac{1}{2}\left(u_{i, j}+u_{j, i}\right)+\Phi_{i j k, k} .
$$

Moreover, the clamping conditions on $\partial \Omega$ read

$$
\underline{\boldsymbol{u}} \stackrel{s}{\otimes} \underline{\boldsymbol{n}}+\underline{\Phi} \cdot \underline{\boldsymbol{n}}=0, \quad \text { i.e., } \quad u_{(i} n_{j)}+\Phi_{i j k} n_{k}=0 \quad \text { on } \partial \Omega,
$$

where $\underline{\boldsymbol{n}}$ is the outer normal vector to $\partial \Omega$.

The purpose of this article is to show the existence and uniqueness of a solution to the above boundary value problem: find $\underline{\boldsymbol{u}}, \underline{\boldsymbol{\Phi}}, \underset{\sim}{\boldsymbol{e}}, \boldsymbol{\sigma}$ and $\underline{\boldsymbol{R}}$ solution to equations (16), (17), (18), as well as the equilibrium equations

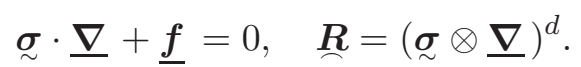

Rather than working with the vector $\underline{\boldsymbol{u}} \in \underline{\mathbb{R}}$ and the deviatoric third rank tensor $\underline{\boldsymbol{\Phi}} \in \mathbb{R}_{S}^{d}$, it is actually more convenient to work with the kinematic variable $\underline{\Psi}=\underline{\Psi}^{s}+\underline{\Psi}^{d} \in \mathbb{R}_{S}$ 
defined as follows. We set $\underline{\Psi}^{d}$, the deviatoric part of $\underline{\boldsymbol{\Psi}}$, to be equal to $\underline{\boldsymbol{\Phi}}$, and we define its spherical part $\underline{\Psi}^{s}$ by

$$
\Psi_{i j k}^{s}=\frac{1}{2}\left(\delta_{i k} u_{j}+\delta_{j k} u_{i}\right) .
$$

We have the remarkable properties

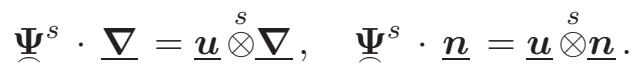

Conversely, for any given $\underline{\Psi} \in \mathbb{R}$, we can extract a unique vector $\underline{\boldsymbol{u}}^{\Psi} \in \underline{\mathbb{R}}$ from its spherical part such that (20) holds true. This vector $\underline{\boldsymbol{u}}^{\Psi}$ is given by

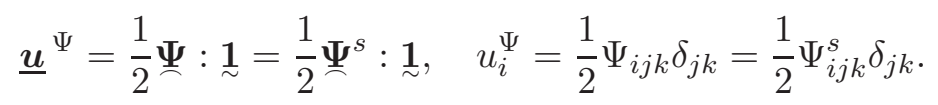

Note that

$$
\frac{1}{2} \underline{\Psi}^{s} \therefore \underline{\Psi}^{s}=\underline{\boldsymbol{u}}^{\Psi} \cdot \underline{\boldsymbol{u}}^{\Psi}
$$

Then, it is clear from (17) that

$$
\underset{\sim}{\boldsymbol{e}}=\underline{\boldsymbol{\Psi}} \cdot \underline{\boldsymbol{\nabla}}, \quad e_{i j}=\Psi_{i j k, k}, \quad \text { and } \quad \underline{\boldsymbol{\Phi}}=\underline{\mathbf{\Psi}}^{d} .
$$

In turn, (18) implies that the clamping boundary conditions on $\partial \Omega$ read

$$
\underline{\Psi} \cdot \underline{\boldsymbol{n}}=0, \quad \Psi_{i j k} n_{k}=0 .
$$

Hence, with this new kinematic variable $\boldsymbol{\Psi}$, which we call the generalized displacement in the sequel, the boundary value problem consists in finding $\underline{\Psi}, \boldsymbol{e}, \boldsymbol{\Phi}, \boldsymbol{\sigma}$ and $\boldsymbol{R}$ solutions to (16), (19), (24) and (25). The existence and uniqueness of a solution to that problem will be shown by Theorem 7 below, that we prove by minimizing an appropriate potential energy (namely, (30) below) over a set of kinematically compatible generalized displacements.

To build that potential energy, it is useful to introduce the Legendre transform $w$ of $w^{*}$, which is defined as

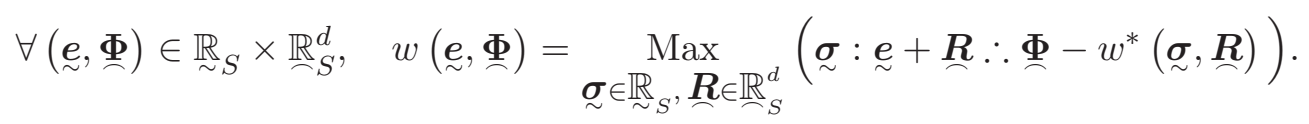

We have assumed that $w^{*}$ is given by (9). We hence have that

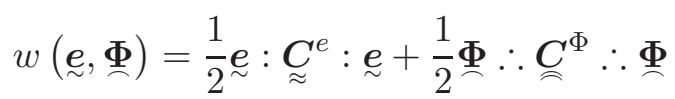

where $\underset{\approx}{\boldsymbol{C}}=(\underset{\approx}{\boldsymbol{S}})^{-1}$ and ${\underset{\Im}{\boldsymbol{\tau}}}^{\Phi}=\left(\underset{\approx}{\boldsymbol{S}}{ }^{R}\right)^{-1}$ are the so-called stiffness tensors. They are symmetric, definite and positive, in the sense that there exists $\beta>0$ such that

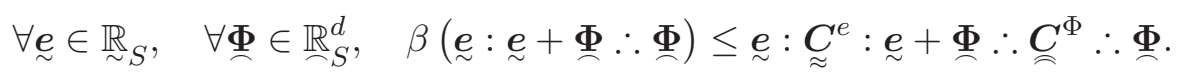


We can then express the generalized stresses $(\underset{\sim}{\boldsymbol{\sigma}}, \underline{\boldsymbol{R}})$ in terms of the generalized strains $(\underset{\sim}{e}, \Phi)$ as

$$
\underset{\sim}{\boldsymbol{\sigma}}=\frac{\partial w}{\partial \underset{\sim}{\boldsymbol{e}}}(\underset{\sim}{\boldsymbol{e}}, \boldsymbol{\Phi}) \quad \text { and } \quad \underline{\boldsymbol{R}}=\frac{\partial w}{\partial \underline{\boldsymbol{\Phi}}}(\underset{\sim}{\boldsymbol{e}}, \underline{\mathbf{\Phi}})
$$

hence, in view of the specific form (26),

$$
\underset{\sim}{\boldsymbol{\sigma}}=\underset{\approx}{\boldsymbol{C}}: \underset{\sim}{\boldsymbol{e}} \quad \text { and } \quad \underline{\boldsymbol{R}}=\stackrel{\boldsymbol{C}}{\Phi}^{\Phi} \therefore \underline{\Phi}
$$

\section{Minimum of the potential energy with clamping boundary conditions}

In this section, we introduce a displacement formulation in the form of the minimization of a potential energy over an appropriate space of kinematically compatible displacement fields (see (34) below). In Theorem 6, we next show that the minimization problem is well-posed.

\subsection{Formulation of the problem}

Let $\underline{\Psi}$ be a generalized displacement field defined over $\Omega$. We know from physical considerations that the potential energy $\mathcal{P}(\underline{\Psi})$ is equal to the strain energy of $\underline{\Psi}$ minus the work of the external load $\underline{\boldsymbol{f}}$. Hence, we have

$$
\mathcal{P}(\underline{\Psi})=\int_{\Omega} w\left(\underline{\Psi} \cdot \underline{\nabla}, \underline{\Psi}^{d}\right)-\int_{\Omega} \frac{1}{2} \underline{f} \cdot(\underline{\Psi}: \underset{\sim}{\mathbf{1}}),
$$

where $w$ is given by (26). Recall that $\underline{\boldsymbol{f}} \in \underline{\boldsymbol{L}}^{2}(\Omega)$. Clearly, $\mathcal{P}(\underline{\Psi})$ is well defined as soon as $\Psi \in K C$, where $K C$ is the space of kinematically compatible generalized displacement fields defined by

$$
K C=\left\{\underline{\Psi} \in \underline{\boldsymbol{L}}_{S}^{2}(\Omega) ; \quad \underline{\boldsymbol{\Psi}} \cdot \underline{\boldsymbol{\nabla}} \in \underset{\sim}{\boldsymbol{L}}{ }_{S}^{2}(\Omega)\right\} .
$$

Lemma 4. The vector space $K C$, endowed with the scalar product

$$
\left\langle\underline{\Psi}_{1}, \underline{\Psi}_{2}\right\rangle_{K C}=\int_{\Omega}\left(\underline{\Psi}_{1} \cdot \underline{\nabla}\right):\left(\underline{\Psi}_{2} \cdot \underline{\nabla}\right)+\underline{\Psi}_{1} \therefore \underline{\Psi}_{2},
$$

is a Hilbert space.

Proof. Again, the proof is based on standard arguments. The application (31) is obviously bilinear, symmetric, definite and positive. Hence, it is a scalar product. The associated norm is denoted $\|\cdot\|_{K C}$. Let us prove that $\left(K C,\|\cdot\|_{K C}\right)$ is complete.

Let $\underline{\Psi}_{n} \in K C, n \in \mathbb{N}$, be a Cauchy sequence in the norm $\|\cdot\|_{K C}$. Then, $\left(\underline{\Psi}_{n}\right)$ is a Cauchy sequence in $\underline{L}_{S}^{2}(\Omega)$ and $\left(\underline{\Psi}_{n} \cdot \underline{\boldsymbol{\nabla}}\right)$ is a Cauchy sequence in ${\underset{\sim}{S}}_{S}^{2}(\Omega)$. Therefore, there exists $\underline{\boldsymbol{\Psi}}_{0} \in \underline{\boldsymbol{L}}_{S}^{2}(\Omega)$ and $\boldsymbol{e}_{0} \in{\underset{\sim}{\boldsymbol{L}}}_{S}^{2}(\Omega)$ such that $\underline{\boldsymbol{\Psi}}_{n}$ and $\underline{\boldsymbol{\Psi}}_{n} \cdot \underline{\boldsymbol{\nabla}}$ converge to $\underline{\boldsymbol{\Psi}}_{0}$ in $\underline{\boldsymbol{L}}_{S}^{2}(\Omega)$ and to $\boldsymbol{e}_{0}$ in $\underset{\sim}{\boldsymbol{L}} \sim_{S}^{2}(\Omega)$, respectively. We thus have that $\underline{\Psi}_{n}$ converges to $\underline{\Psi}_{0}$ in $\boldsymbol{D}_{S}^{\prime}(\Omega)$, hence $\underline{\Psi}_{n} \cdot \underline{\boldsymbol{\nabla}}$ converges to $\underline{\Psi}_{0} \cdot \underline{\boldsymbol{\nabla}}$ in $\underline{\sim}_{S}^{\prime}(\Omega)$. We also have that $\underline{\boldsymbol{\Psi}}_{n} \cdot \underline{\boldsymbol{\nabla}}$ converges to ${\underset{\sim}{\boldsymbol{e}}}_{0}$ in $\widetilde{D}_{S}^{\prime}(\bar{\Omega})$. Therefore, $\underset{\sim}{\boldsymbol{e}_{0}}=\underline{\boldsymbol{\Psi}}_{0} \cdot \underline{\boldsymbol{\nabla}}$. This proves that $\underline{\Psi}_{0} \in K C$ and that $\underline{\Psi}_{n}$ converges to $\underline{\Psi}_{0}$ according to the norm $\|\cdot\|_{K C}$. 
We now give a rigorous sense to the boundary condition (25). Recall indeed that the trace on $\partial \Omega$ of $\underline{\Psi} \in K C$ is not defined, as $\underline{\Psi}$ does not belong to $\boldsymbol{H}_{S}^{1}(\Omega)$. However, the trace of $\underline{\boldsymbol{\Psi}} \cdot \underline{\boldsymbol{n}}$ can be defined. For smooth fields $\underline{\boldsymbol{\Psi}}$ and $\underset{\sim}{\boldsymbol{\varphi}}$ in $\boldsymbol{C}_{S}^{\infty}(\Omega)$, we have

$$
\int_{\Omega} \underset{\sim}{\varphi}:(\underline{\Psi} \cdot \underline{\nabla})+(\underset{\sim}{\varphi} \otimes \underline{\nabla}) \therefore \underline{\Psi}=\int_{\partial \Omega} \underset{\sim}{\varphi}:(\underline{\Psi} \cdot \underline{\boldsymbol{n}}) .
$$

Because $\underline{\varphi}$ can be arbitrary prescribed on $\partial \Omega$, imposing $\underline{\boldsymbol{\Psi}} \cdot \underline{\boldsymbol{n}}=0$ on $\partial \Omega$ is equivalent to imposing

$$
\int_{\Omega} \underset{\sim}{\varphi}:(\underline{\Psi} \cdot \underline{\nabla})+(\underset{\sim}{\varphi} \otimes \underline{\nabla}) \therefore \underline{\Psi}=0
$$

for all $\underset{\sim}{\boldsymbol{\sim}} \in{\underset{\sim}{S}}_{S}^{\infty}(\Omega)$. We thus define $K C_{0} \subset K C$, the subspace of generalized displacements which are kinematically compatible with clamping boundary conditions, as

$$
K C_{0}=\left\{\underline{\Psi} \in K C ; \quad(33) \text { holds true for all } \underset{\sim}{\boldsymbol{\varphi}} \in \underset{\sim}{\boldsymbol{H}}{ }_{S}^{1}(\Omega)\right\} .
$$

It is readily seen that the subspace $K C_{0}$ is closed in $K C$ equipped with its norm $\|\cdot\|_{K C}$. Hence, $K C_{0}$, endowed with the scalar product $\langle\cdot, \cdot\rangle_{K C}$, is a Hilbert space.

The stress gradient problem with clamped boundary conditions consists in minimizing the potential energy $\mathcal{P}(\underline{\Psi})$ over all $\underline{\Psi} \in K C_{0}$ :

$$
\inf \left\{\mathcal{P}(\underline{\Psi}), \quad \underline{\Psi} \in K C_{0}\right\}
$$

where $\mathcal{P}(\underline{\Psi})$ is defined by (30). Note that

$$
\mathcal{P}(\underline{\Psi})=\frac{1}{2} a(\underline{\Psi}, \underline{\Psi})-b(\underline{\Psi}),
$$

where the bilinear form $a$ is defined on $K C \times K C$ by

$$
a\left(\underline{\Psi}_{1}, \underline{\Psi}_{2}\right)=\int_{\Omega}\left(\underline{\Psi}_{1} \cdot \underline{\nabla}\right):{\underset{\approx}{C}}^{e}:\left(\underline{\Psi}_{2} \cdot \underline{\nabla}\right)+\underline{\Psi}_{1}^{d} \therefore \stackrel{C}{\Phi}^{\Phi} \therefore \underline{\Psi}_{2}^{d},
$$

and the linear form $b$ is defined on $K C$ by

$$
b(\underline{\Psi})=\frac{1}{2} \int_{\Omega} \underline{f} \cdot(\underline{\Psi}: \underset{\sim}{\mathbf{1}}) .
$$

Obviously, $b$ is a continuous linear form on $K C$ and $a$ is a continuous symmetric bilinear form on $K C \times K C$. To show that (34) is well-posed, we are thus left with showing that the bilinear form $a$ is coercive on $K C_{0}$. The difficulty comes from the fact that only the deviatoric part $\underline{\Psi}^{d}$ of $\underline{\Psi}$ appears in $a$, while the norm $\|\cdot\|_{K C}$ is written in terms of the full tensor $\underline{\Psi}$. 


\subsection{Coercivity of the bilinear form a}

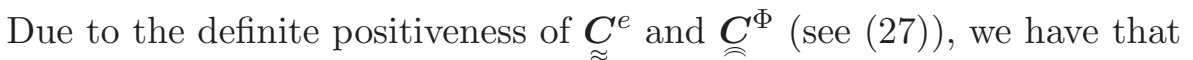

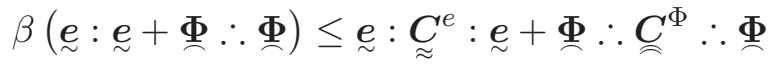

for all $\underset{\sim}{e} \in \mathbb{\sim}_{\sim}$ and all $\underset{\Phi}{\Phi}{\underset{\mathbb{R}}{S}}_{S}^{d}$, where $\beta>0$. Introduce the vector space $K C^{d}$ defined by

$$
K C^{d}=\left\{\underline{\Psi} \in \underline{D}_{S}^{\prime}(\Omega) ; \quad \underline{\Psi}^{d} \in \underline{\sim}_{S}^{2}(\Omega) ; \quad \underline{\Psi} \cdot \underline{\boldsymbol{\nabla}} \in{\underset{\sim}{\boldsymbol{L}}}_{S}^{2}(\Omega)\right\},
$$

which differs from $K C$ by the fact that we do not assume that the spherical component $\underline{\Psi}^{s}$ belongs to $\underline{L}_{S}^{2}(\Omega)$. We endow $K C^{d}$ with the scalar product

$$
\left\langle\underline{\Psi}_{1}, \underline{\Psi}_{2}\right\rangle_{K C^{d}}=\int_{\Omega}\left(\underline{\Psi}_{1} \cdot \underline{\nabla}\right):\left(\underline{\Psi}_{2} \cdot \underline{\nabla}\right)+\underline{\Psi}_{1}^{d} \therefore \underline{\Psi}_{2}^{d}
$$

and the induced norm

$$
\|\underline{\Psi}\|_{K C^{d}}^{2}=\langle\underline{\Psi}, \underline{\Psi}\rangle_{K C^{d}} .
$$

We have that $K C \subset K C^{d}$ and that

$$
\forall \underline{\Psi} \in K C, \quad \beta\|\underline{\Psi}\|_{K C^{d}}^{2} \leq a(\underline{\Psi}, \underline{\Psi}) .
$$

The coercivity of $a$ over $K C_{0}$ equipped with the norm $\|\cdot\|_{K C}$ is a direct consequence of the following result:

Lemma 5. We have that $K C^{d}=K C$ and $\|\cdot\|_{K C^{d}}$ defines a norm on $K C_{0}$ which is equivalent to the norm $\|\cdot\|_{K C}$.

Proof. The proof falls in three steps.

Step 1. Let $\Psi \in K C^{d}$. We prove that the spherical part of $\boldsymbol{\Psi}$ is in $\underline{L}_{S}^{2}(\Omega)$. To that aim, consider the vector field $\underline{\boldsymbol{u}}^{\Psi} \in \underline{\boldsymbol{D}}^{\prime}(\Omega)$ defined by (22). Then,

$$
\underline{\boldsymbol{u}}^{\Psi} \stackrel{s}{\otimes} \underline{\boldsymbol{\nabla}}=\underline{\Psi}^{s} \cdot \underline{\boldsymbol{\nabla}}=(\underline{\Psi} \cdot \underline{\boldsymbol{\nabla}})-\left(\underline{\Psi}^{d} \cdot \underline{\boldsymbol{\nabla}}\right)
$$

is in $\boldsymbol{H}_{S}^{-1}(\Omega)$ because, by definition of $K C^{d}$, we have that $\underline{\boldsymbol{\Psi}} \cdot \underline{\boldsymbol{\nabla}} \in{\underset{\sim}{\boldsymbol{L}}}_{S}^{2}(\Omega)$ and $\underline{\mathbf{\Psi}}^{d} \in \underline{\boldsymbol{L}}_{S}^{2}(\Omega)$. Therefore, according to (Amrouche et al., 2006, Theorem 3.1), $\underline{\boldsymbol{u}}^{\Psi}$ is in $\underline{\boldsymbol{L}}^{2}(\Omega)$. Hence, in view of (23), we obtain that $\underline{\Psi}^{s} \in \underline{L}_{S}^{2}(\Omega)$. This proves that $\underline{\Psi} \in K C$, thus $K C^{d} \subset K C$. The converse inclusion being obvious, we have that $K C^{d}=K C$.

Note that

$$
\forall \underline{\Psi} \in K C, \quad\|\underline{\Psi}\|_{K C}^{2}=\|\underline{\Psi}\|_{K C^{d}}^{2}+\left\|\underline{\Psi}^{s}\right\|_{\sim}^{2}{\underset{\sim}{S}(\Omega)}_{2}^{2} \geq\|\underline{\Psi}\|_{K C^{d}}^{2} .
$$

Step 2. We now show that the positive symmetric bilinear form $\langle\cdot, \cdot\rangle_{K C^{d}}$ is definite on $K C_{0}$, and hence it is a scalar product on $K C_{0}$. Let $\underline{\Psi} \in K C_{0}$ such that $\|\underline{\Psi}\|_{K C^{d}}=0$. Then $\underline{\Psi}^{d}=0$ and $\underline{\boldsymbol{\Psi}} \cdot \underline{\boldsymbol{\nabla}}=0$. From (38), we get that $\underline{\boldsymbol{u}}^{\Psi} \stackrel{s}{\otimes} \underline{\boldsymbol{\nabla}}=0$. Therefore, $\underline{\boldsymbol{u}}^{\Psi}$ is a rigid 
body displacement field of the form (5) (see (Moreau, 1979)). We hence infer from (20) that $\Psi^{s}$ is smooth.

In addition, since $\Psi \in K C_{0}$, it satisfies (33), which reads, taking into account that $\underline{\Psi}^{d}=0$ and $\underline{\Psi} \cdot \underline{\nabla}=0$ :

$$
\forall \underset{\sim}{\boldsymbol{\varphi}} \in \underset{\sim}{\boldsymbol{H}}{ }_{S}^{1}(\Omega), \quad \int_{\Omega}(\underset{\sim}{\boldsymbol{\varphi}} \otimes \underline{\boldsymbol{\nabla}}) \therefore \underline{\Psi}^{s}=0
$$

Since $\underline{\Psi}^{s}$ is smooth, we can integrate by part in the above expression. This yields, in view of (32), that

$$
\forall \underline{\sim} \in{\underset{\sim}{H}}_{S}^{1}(\Omega), \quad \int_{\Omega} \underset{\sim}{\varphi}:\left(\underline{\Psi}^{s} \cdot \underline{\nabla}\right)=\int_{\partial \Omega} \underset{\sim}{\varphi}:\left(\underline{\Psi}^{s} \cdot \underline{\boldsymbol{n}}\right) .
$$

Using (21), we have $\underline{\boldsymbol{\Psi}}^{s} \cdot \underline{\boldsymbol{\nabla}}=\underline{\boldsymbol{u}}^{\Psi} \stackrel{s}{\otimes} \underline{\boldsymbol{\nabla}}=0$ and $\underline{\boldsymbol{\Psi}}^{s} \cdot \underline{\boldsymbol{n}}=\underline{\boldsymbol{u}}^{\Psi} \stackrel{s}{\otimes \boldsymbol{n}}$. We hence deduce that, for any $\underset{\sim}{\boldsymbol{\varphi}} \in \underset{\sim}{\boldsymbol{H}} \boldsymbol{\sim}_{S}^{1}(\Omega)$,

$$
\begin{aligned}
0=\int_{\partial \Omega} \underset{\sim}{\varphi}:\left(\underline{\boldsymbol{u}}^{\Psi} \stackrel{s}{\otimes} \underline{\boldsymbol{n}}\right)=\int_{\partial \Omega}(\underset{\sim}{\boldsymbol{\varphi}} \cdot \underline{\boldsymbol{n}}) \cdot \underline{\boldsymbol{u}}^{\Psi}=\int_{\partial \Omega}(\underset{\sim}{\boldsymbol{\varphi}} \cdot \underline{\boldsymbol{n}}) \cdot(\underline{\boldsymbol{t}}+\underline{\boldsymbol{\omega}} \times \underline{\boldsymbol{x}}) \\
=\left[\int_{\partial \Omega} \underset{\sim}{\boldsymbol{\varphi}} \cdot \underline{\boldsymbol{n}}\right] \cdot \underline{\boldsymbol{t}}+\left[\int_{\partial \Omega} \underline{\boldsymbol{x}} \times(\underline{\sim} \cdot \underline{\boldsymbol{n}})\right] \cdot \underline{\boldsymbol{\omega}} .
\end{aligned}
$$

The tensor $\varphi$ being arbitrary, we obtain that $\underline{\boldsymbol{t}}=\underline{\boldsymbol{\omega}}=0$. This implies that $\underline{\boldsymbol{u}}^{\Psi}=0$, hence $\underline{\Psi}^{s}=0$, hence $\underline{\Psi}=0$. We have thus shown that, if $\underline{\boldsymbol{\Psi}} \in K C_{0}$ satisfies $\|\underline{\boldsymbol{\Psi}}\|_{K C^{d}}=0$, then $\Psi=0$.

Step 3. We claim that there exists a constant $C^{\prime}>0$ such that

$$
\forall \underline{\Psi} \in K C_{0}, \quad\|\underline{\Psi}\|_{K C} \leq C^{\prime}\|\underline{\Psi}\|_{K C^{d}} .
$$

To prove this claim, we proceed by contradiction. If (40) does not hold, then, for all $k \in \mathbb{N}$, there exists $\underline{\Psi}_{k} \in K C_{0}$ such that

$$
\left\|\underline{\Psi}_{k}\right\|_{K C}=1, \quad\left\|\underline{\Psi}_{k}\right\|_{K C^{d}} \leq \frac{1}{k} .
$$

We therefore have $\left\|\underline{\boldsymbol{\Psi}}_{k} \cdot \underline{\boldsymbol{\nabla}}\right\|_{\boldsymbol{L}_{S}^{2}(\Omega)} \leq 1 / k$. In addition, we have $\left\|\underline{\Psi}_{k}^{d}\right\|_{\underline{\boldsymbol{L}}_{S}^{2}(\Omega)} \leq 1 / k$, which implies that $\left\|\underline{\Psi}_{k}^{d} \cdot \underline{\boldsymbol{\nabla}}\right\|_{\boldsymbol{H}_{S}^{-1}(\Omega)} \leq 1 / k$

Consider the sequence of vector fields $\underline{\boldsymbol{u}}_{k}$ defined from $\underline{\Psi}_{k}$ by (22). We know that the corresponding sequence of strain fields, which is given by (38), satisfies $\| \underline{\boldsymbol{u}}_{k} \stackrel{\stackrel{s}{\otimes} \boldsymbol{\nabla}}{\|}{\underset{\sim}{\boldsymbol{H}_{S}^{-1}(\Omega)}} \leq$ $2 / k$. In view of $(7)$, we deduce that

$$
\inf _{\underline{\boldsymbol{r}} \in \underline{\mathcal{R}}}\left\|\underline{\boldsymbol{u}}_{k}-\underline{\boldsymbol{r}}\right\|_{\underline{\boldsymbol{L}}^{2}(\Omega)} \leq \frac{C}{k}
$$


We now point out that the above infimum is actually attained at a unique $\underline{\boldsymbol{r}}_{k} \in \underline{\mathcal{R}}$, which simply is the orthogonal projection of $\underline{\boldsymbol{u}}_{k}$ on $\underline{\mathcal{R}}$ in the sense of the scalar product $(\cdot, \cdot) \underline{\boldsymbol{L}}_{(\Omega)}{ }^{(\Omega)}$. Hence, $\left\|\underline{\boldsymbol{r}}_{k}\right\|_{\underline{\boldsymbol{L}}^{2}(\Omega)} \leq\left\|\underline{\boldsymbol{u}}_{k}\right\|_{\underline{\boldsymbol{L}}^{2}(\Omega)}$, which is bounded in $\underline{\boldsymbol{L}}^{2}(\Omega)$ because we have $\left\|\underline{\Psi}_{k}\right\|_{K C}=1$. Consequently, the sequence $\left(\underline{\boldsymbol{r}}_{k}\right)$ is also bounded in the six-dimensional space $\underline{\mathcal{R}}$ of rigid body displacements. Thus, there exists a subsequence $\left(\underline{\boldsymbol{r}}_{l}\right)$ which strongly converges in $\underline{\boldsymbol{L}}^{2}(\Omega)$ to some $\underline{\boldsymbol{r}}_{0} \in \underline{\boldsymbol{\mathcal { R }}}$. In view of (41), we write

$$
\left\|\underline{\boldsymbol{u}}_{l}-\underline{\boldsymbol{r}}_{l}\right\|_{\underline{\boldsymbol{L}}^{2}(\Omega)}=\inf _{\underline{\boldsymbol{r}} \in \underline{\mathcal{R}}}\left\|\underline{\boldsymbol{u}}_{l}-\underline{\boldsymbol{r}}\right\|_{\underline{\boldsymbol{L}}^{2}(\Omega)} \leq \frac{C}{l},
$$

which implies that the subsequence $\left(\underline{\boldsymbol{u}}_{l}\right)$ strongly converges in $\underline{\boldsymbol{L}}^{2}(\Omega)$ to $\underline{\boldsymbol{r}}_{0} \in \underline{\mathcal{R}}$. Therefore, $\left(\underline{\Psi}_{l}\right)$ strongly converges in $K C$ to some element $\underline{\Psi}_{0}$ satisfying $\left\|\underline{\Psi}_{0}\right\|_{K C^{d}}=0$. In addition, $\underline{\Psi}_{k} \in K C_{0}$ for all $k$, hence $\underline{\Psi}_{0} \in K C_{0}$. In view of the conclusion of Step 2, this implies that $\underline{\Psi}_{0}=0$. This is a contradiction with the fact that $\left\|\Psi_{l}\right\|_{K C}=1$ for all $l$ and that $\underline{\Psi}_{l}$ strongly converges in $K C$ to $\underline{\Psi}_{0}$. This concludes the proof of the claim (40).

The equivalence of the norms $\|\cdot\|_{K C^{d}}$ and $\|\cdot\|_{K C}$ on $K C_{0}$ is a direct consequence of (39) and (40).

\subsection{Existence and uniqueness of the solution to (34)}

The symmetric bilinear form $a$ being continuous and coercive on the Hilbert space $K C_{0}$ endowed with the scalar product $\langle\cdot, \cdot\rangle_{K C}$, and the linear form $b$ being continuous on this space, we obtain the following result as a consequence of the Lax-Milgram theorem:

Theorem 6. Assume that $\underline{\boldsymbol{f}} \in \underline{\boldsymbol{L}}^{2}(\Omega)$ and that the stiffness tensors $\underset{\approx}{\boldsymbol{C}}$ and $\boldsymbol{C}^{\Phi}$ are symmetric and positive definite in the sense of (27). Then the minimization problem (34) admits a unique solution $\underline{\Psi}^{\#}$. It is also the unique solution of the following problem: find $\underline{\Psi}^{\#}$ in $K C_{0}$ such that $a\left(\underline{\mathbf{\Psi}}^{\#}, \underline{\Psi}\right)=b(\underline{\mathbf{\Psi}})$ for all $\underline{\boldsymbol{\Psi}}$ in $K C_{0}$, where $a$ and $b$ are defined by (35) and (36).

\section{Relation between the stress and the displacement formulations}

We have considered above a stress formulation of the problem, namely (13). As shown by Theorem 3, this problem has a unique solution. We next have considered the displacement formulation (34), which also has a unique solution, in view of Theorem 6 . We now show that one can build the solution of the former using the solution of the latter.

Theorem 7. Under the assumptions of Theorem 6, let $\underline{\Psi}^{\#}$ be the unique solution to the minimization problem (34). Let

$$
\stackrel{e}{ }^{\#}=\underline{\Psi}^{\#} \cdot \underline{\nabla} \quad \text { and } \quad \underline{\Phi}^{\#}=\left(\underline{\Psi}^{\#}\right)^{d}
$$

be the generalized strain fields associated to $\underline{\Psi}^{\#}$ by the compatibility conditions (24) and let

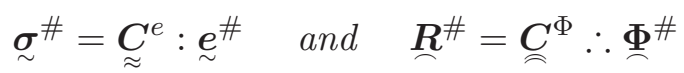


be the generalized stress fields associated to the generalized strain fields by the constitutive law (29).

Then $\boldsymbol{\sigma}^{\#}$ is in the space $S A(\underline{\boldsymbol{f}})$ defined by $(12)$ while $\underline{\boldsymbol{R}}^{\#}=\left(\boldsymbol{\sigma}^{\#} \otimes \underline{\boldsymbol{\nabla}}\right)^{d}$. In addition, $\boldsymbol{\sigma}^{\#}$ is the unique solution to the stress formulation problem (13), where $w^{*}$ is defined by (9).

Proof. By construction, we have that ${\underset{\sim}{\boldsymbol{e}}}^{\#} \in \underset{\sim}{\boldsymbol{L}}{ }_{S}^{2}(\Omega), \underline{\boldsymbol{\Phi}}^{\#} \in \sim_{S}^{\boldsymbol{L}}(\Omega), \boldsymbol{\sigma}^{\#} \in \underset{\sim}{\boldsymbol{L}}{ }_{S}^{2}(\Omega)$ and $\underline{\boldsymbol{R}}^{\#} \in \underline{L}_{S}^{2}(\Omega)$. According to the variational equation satisfied by $\underline{\Psi}^{\#}$ (see Theorem 6 ), we have that, for all $\underline{\Psi} \in K C_{0}$,

$$
\int_{\Omega} \boldsymbol{\sigma}^{\#}:(\underline{\Psi} \cdot \underline{\nabla})+\underline{\boldsymbol{R}}^{\#} \therefore \underline{\Psi}^{d}=\int_{\Omega} \frac{1}{2} \underline{f} \cdot(\underline{\Psi}: \underset{\sim}{\mathbf{1}}) .
$$

Restricting the above relation to $\underline{\Psi} \in \underline{D}_{S}(\Omega) \subset K C_{0}$, we obtain

$$
\forall \underline{\Psi} \in \underline{\boldsymbol{D}}_{S}(\Omega), \quad\left\langle\left(\underline{\boldsymbol{R}}^{\#}-\underline{\boldsymbol{F}}^{s}-\underline{\boldsymbol{\sigma}}^{\#} \otimes \underline{\boldsymbol{\nabla}}\right), \underline{\boldsymbol{\Psi}}\right\rangle_{\underline{\boldsymbol{D}}_{S}^{\prime}(\Omega), \underline{\boldsymbol{D}}_{S}(\Omega)}=0,
$$

where we have used the fact that $\underline{\boldsymbol{R}}^{\#}$ is deviatoric to substitute $\underline{\boldsymbol{R}}^{\#} \therefore \underline{\boldsymbol{\Psi}}$ for $\underline{\boldsymbol{R}}^{\#} \therefore \underline{\boldsymbol{\Psi}}^{d}$, and where $\boldsymbol{F}^{s} \in \mathbb{R}_{S}^{s}$ is defined by

$$
F_{i j k}^{s}=\frac{1}{4}\left(\delta_{i k} f_{j}+\delta_{j k} f_{i}\right),
$$

so that $\underline{\boldsymbol{F}}^{s} \therefore \Psi=\frac{1}{2} \underline{\boldsymbol{f}} \cdot(\underline{\Psi}: \underset{\sim}{\mathbf{1}})$. Hence, we have shown that

$$
\stackrel{\sim}{\sim}^{\#} \otimes \underline{\boldsymbol{\nabla}}=\underline{\boldsymbol{R}}^{\#}-\underline{\boldsymbol{F}}^{s}
$$

in the sense of distributions. Taking the spherical part of (43), we obtain

$$
\left(\stackrel{\boldsymbol{\sigma}}{\#}^{\#} \otimes \underline{\boldsymbol{\nabla}}\right)^{s}=-\underline{\boldsymbol{F}}^{s}
$$

since $\boldsymbol{F}^{s}$ is spherical by construction, while $\underline{\boldsymbol{R}}^{\#}$ is deviatoric. Using (3), we deduce that

$$
\underline{\sim}^{\#} \cdot \underline{\nabla}=-\underline{F}^{s}: \underset{\sim}{1}=-\underline{\boldsymbol{f}} .
$$

Taking now the deviatoric part of (43), we obtain

$$
\left(\stackrel{\boldsymbol{\sigma}}{\#}^{\#} \otimes \underline{\boldsymbol{\nabla}}\right)^{d}=\underline{\boldsymbol{R}}^{\#} .
$$

Moreover, the equation (43) shows that the stress gradient components are in $L^{2}(\Omega)$. We therefore obtain that $\underline{\sim}^{\#} \in S A(\underline{\boldsymbol{f}})$.

We now show that $\boldsymbol{\sigma}^{\#}$ is the unique solution to the stress formulation problem (13). Let $\underset{\sim}{\boldsymbol{\sigma}}$ be any element in $S A(\underline{\boldsymbol{f}})$, and set $\boldsymbol{\sigma}^{\prime}=\underset{\sim}{\boldsymbol{\sigma}}-\boldsymbol{\sigma}^{\#}$. Then, by simple algebra, we have

$$
\begin{aligned}
& \mathcal{P}^{*}(\underset{\sim}{\boldsymbol{\sigma}})=\mathcal{P}^{*}\left({\underset{\sim}{\boldsymbol{\sigma}}}^{\#}\right)+\mathcal{P}^{*}\left(\boldsymbol{\sigma}^{\prime}\right)+\int_{\Omega}{\underset{\sim}{\boldsymbol{\sigma}}}^{\prime}: \underset{\sim}{\boldsymbol{S}^{\sigma}}:{\underset{\sim}{\boldsymbol{\sigma}^{\#}}+\left(\boldsymbol{\sigma}^{\prime} \otimes \underline{\boldsymbol{\nabla}}\right.}^{d} \therefore \underline{\sim}^{R} \therefore\left(\boldsymbol{\sigma}^{\#} \otimes \underline{\boldsymbol{\nabla}}\right)^{d} \\
& =\mathcal{P}^{*}\left(\underline{\boldsymbol{\sigma}}^{\#}\right)+\mathcal{P}^{*}\left(\boldsymbol{\sigma}^{\prime}\right)+\int_{\Omega} \underline{\sim}^{\prime}:\left(\underline{\boldsymbol{\Psi}}^{\#} \cdot \underline{\boldsymbol{\nabla}}\right)+\left(\underline{\boldsymbol{\sigma}}^{\prime} \otimes \underline{\boldsymbol{\nabla}}\right)^{d} \therefore\left(\underline{\Psi}^{\#}\right)^{d},
\end{aligned}
$$


where we have used that $\underline{\Psi}^{\#} \cdot \underline{\boldsymbol{\nabla}}={\underset{\sim}{\boldsymbol{e}}}^{\#}={\underset{\sim}{\boldsymbol{S}}}^{\sigma}: \boldsymbol{\sigma}^{\#}$ and $\left(\underline{\boldsymbol{\Psi}}^{\#}\right)^{d}=\underline{\boldsymbol{\Phi}}^{\#}=\underline{\boldsymbol{S}}^{R} \therefore \underline{\boldsymbol{R}}^{\#}=\underline{\boldsymbol{S}}^{R} \therefore$ $\left(\boldsymbol{\sigma}^{\#} \otimes \underline{\boldsymbol{\nabla}}\right)^{d}$. Observe that

$$
\left(\stackrel{\boldsymbol{\sigma}}{\prime}^{\prime} \otimes \underline{\boldsymbol{\nabla}}\right)^{d} \therefore\left(\underline{\boldsymbol{\Psi}}^{\#}\right)^{d}=\left(\underline{\boldsymbol{\sigma}}^{\prime} \otimes \underline{\boldsymbol{\nabla}}\right)^{d} \therefore \underline{\boldsymbol{\Psi}}^{\#}=\left(\underline{\boldsymbol{\sigma}}^{\prime} \otimes \underline{\boldsymbol{\nabla}}\right) \therefore \underline{\Psi}^{\#},
$$

the last equality being a consequence of the fact that $\underline{\sim}^{\prime} \in S A(\underline{\mathbf{0}})$. We hence get that

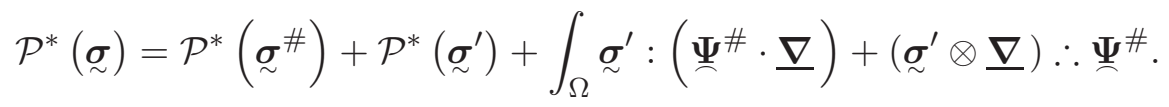

The generalized displacement field $\underline{\Psi}^{\#}$ belongs to $K C_{0}$, hence satisfies (33), and therefore the last term in the above equation vanishes. We obtain that

$$
\mathcal{P}^{*}(\underset{\sim}{\boldsymbol{\sigma}})=\mathcal{P}^{*}\left({\underset{\sim}{\boldsymbol{\sigma}}}^{\#}\right)+\mathcal{P}^{*}\left(\boldsymbol{\sigma}^{\prime}\right)
$$

and we eventually note that $\mathcal{P}^{*}\left(\underset{\sim}{\boldsymbol{\sigma}^{\prime}}\right)$ is positive as soon as $\boldsymbol{\sigma}_{\sim}^{\prime}$ does not vanish. Thus $\boldsymbol{\sigma}^{\#} \in S A(\underline{\boldsymbol{f}})$ is indeed the unique solution to (13).

\section{Minimum of the potential energy with free boundary conditions}

The purpose of this section is to study the problem when the free boundary condition

$$
\underset{\sim}{\boldsymbol{\sigma}}=0 \quad \text { on } \quad \partial \Omega
$$

is substituted to the clamping boundary condition (25). The boundary value problem with free boundary conditions hence consists in finding $\underline{\boldsymbol{\Psi}}, \underset{\sim}{\boldsymbol{e}}, \boldsymbol{\Phi}, \underset{\sim}{\boldsymbol{\sigma}}$ and $\boldsymbol{R}$ solution to (16), (19), (24) and (44).

We first introduce an appropriate displacement formulation (see (48) below) before showing that the corresponding minimization problem is well-posed (Theorem 10). We eventually show the link with a stress formulation in Theorem 12

\subsection{Formulation of the problem}

Because there are no contact forces at the boundary $\partial \Omega$, the applied body forces $\underline{\boldsymbol{f}} \in \underline{\boldsymbol{L}}^{2}(\Omega)$, or their third rank tensor representation $\underline{\boldsymbol{F}}^{s}$ defined by (42), must be selfbalanced, that is

$$
\forall \underline{\Psi} \in \mathcal{R}, \quad b(\underline{\Psi})=\frac{1}{2} \int_{\Omega} \underline{\boldsymbol{f}} \cdot(\underline{\Psi}: \underset{\sim}{\mathbf{1}})=\int_{\Omega} \underline{\boldsymbol{f}} \cdot \underline{\boldsymbol{u}}^{\Psi}=\int_{\Omega} \underline{\boldsymbol{F}}^{s} \therefore \Psi=0 .
$$

This is equivalent to the two standard conditions

$$
\int_{\Omega} \underline{f}=0 \quad \text { and } \quad \int_{\Omega} \underline{x} \times \underline{f}=0 .
$$

We have introduced in (6) the space $\underline{\mathcal{R}}$ of rigid body displacements. We have also seen that we can associate a spherical third rank tensor $\underline{\boldsymbol{\Psi}}$ to any displacement field $\underline{\boldsymbol{u}}$ by (20). 
It is hence natural to introduce the set of spherical third rank tensors that are associated to $\underline{\mathcal{R}}$ :

$$
\underline{\mathcal{R}}=\left\{\underline{\Psi} \in K C ; \quad \Psi_{i j k}=\frac{1}{2}\left(\delta_{i k} u_{j}+\delta_{j k} u_{i}\right) ; \quad \underline{\boldsymbol{u}} \text { is a function of the form (5) }\right\} .
$$

Introduce next the quotient space $\widehat{K C}=K C / \underline{\mathcal{R}}$. All the elements $\underline{\boldsymbol{\Psi}}$ of $\widehat{\boldsymbol{\Psi}} \in \widehat{K C}$ have the same deviatoric part. Their spherical parts differ by an element of $\mathcal{R}$.

The space $\mathcal{R}$ is a closed subspace of the Hilbert space $K C$. We can therefore consider the orthogonal projection of $K C$ on $\mathcal{R}$ according to the scalar product $\langle\cdot, \cdot\rangle_{K C}$ : for any $\underline{\Psi} \in K C$, there exists a unique $\Pi(\Psi) \in \mathcal{R}$ such that

$$
\|\underline{\Psi}-\Pi(\underline{\Psi})\|_{K C}=\inf _{z \in \mathcal{R}}\|\underline{\Psi}-z\|_{K C} .
$$

Lemma 8. The quotient space $\widehat{K C}=K C / \underline{\mathcal{R}}$, endowed with the scalar product

$$
\left\langle\widehat{\Psi}_{1}, \widehat{\Psi}_{2}\right\rangle_{\widehat{K C}}=\left\langle\underline{\Psi}_{1}-\Pi\left(\underline{\Psi}_{1}\right), \underline{\Psi}_{2}-\Pi\left(\underline{\Psi}_{2}\right)\right\rangle_{K C},
$$

where $\underline{\Psi}_{1}$ (resp. $\underline{\Psi}_{2}$ ) is any element in $\widehat{\Psi}_{1}$ (resp. $\widehat{\Psi}_{2}$ ), is a Hilbert space.

Proof. We first show that the scalar product (47) is well-defined. Consider $\underline{\Psi}_{1}$ and $\Psi_{1}^{\prime}$ two elements in $\widehat{\Psi}_{1}$. We see that

$$
\underline{\Psi}_{1}^{\prime}-\Pi\left(\underline{\Psi}_{1}^{\prime}\right)=\left(\underline{\Psi}_{1}^{\prime}-\underline{\Psi}_{1}-\Pi\left(\underline{\Psi}_{1}^{\prime}-\underline{\Psi}_{1}\right)\right)+\underline{\Psi}_{1}-\Pi\left(\underline{\Psi}_{1}\right)
$$

and we recall that $\underline{\Psi}_{1}^{\prime}-\underline{\Psi}_{1}$ is an element of $\mathcal{R}$. The first term in the above right-hand side hence vanishes. This implies that $\Psi_{1}^{\prime}-\Pi\left(\Psi_{1}^{\prime}\right)=\underline{\Psi}_{1}-\Pi\left(\Psi_{1}\right)$ and thus the scalar product (47) is well-defined on $\widehat{K C}$.

We now show that $\widehat{K C}$ is a Hilbert space. Let $\widehat{\Psi}_{n} \in \widehat{K C}, n \in \mathbb{N}$, be a Cauchy sequence in the norm $\|\cdot\|_{\widehat{K C}}$. We thus see that $\Psi_{n}-\Pi\left(\Psi_{n}\right)$ is a Cauchy sequence in $K C$, which thus converges to some $\underline{\Psi}_{\infty}$ satisfying $\Pi\left(\underline{\Psi}_{\infty}\right)=0$ by continuity of the projection $\Pi$. We now observe that

$$
\left\|\widehat{\Psi}_{n}-\widehat{\Psi}_{\infty}\right\|_{\widehat{K C}}^{2}=\left\|\underline{\Psi}_{n}-\underline{\Psi}_{\infty}-\Pi\left(\underline{\Psi}_{n}\right)\right\|_{K C}^{2},
$$

hence $\widehat{\Psi}_{n}$ converges to $\widehat{\Psi}_{\infty}$ in $\widehat{K C}$. This proves that $\widehat{K C}$ is a complete space.

We now introduce a variational formulation on $\widehat{K C}$, inspired by the one established on $K C_{0}$ in Theorem 6 . In the spirit of (36), we introduce on $\widehat{K C}$ the linear form

$$
\forall \widehat{\Psi} \in \widehat{K C}, \quad \widehat{b}(\widehat{\mathbf{\Psi}})=b(\underline{\Psi})=\int_{\Omega} \underline{\boldsymbol{F}}^{s} \therefore \underline{\Psi}^{s}
$$

where $\boldsymbol{\Psi}$ is any element in $\widehat{\underline{\Psi}}$. Because of (45), the above right-hand side does not depend on the choice of $\underline{\Psi}$. Using the continuity of $b$ on $K C$, we see that, for any $\widehat{\Psi} \in \widehat{K C}$,

$$
|\widehat{b}(\widehat{\underline{\Psi}})|=|b(\Psi-\Pi(\underline{\Psi}))| \leq C\|\underline{\Psi}-\Pi(\underline{\Psi})\|_{K C}=C\|\widehat{\Psi}\|_{\widehat{K C}} .
$$


The linear form $\widehat{b}$ is hence continuous on $\widehat{K C}$.

Likewise, in the spirit of (35), we introduce on $\widehat{K C}$ the bilinear form

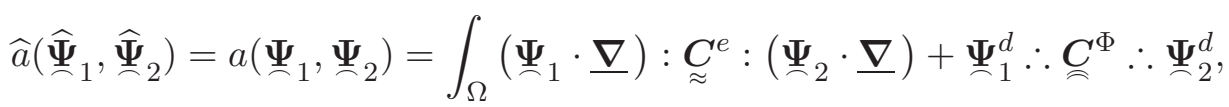

where $\underline{\Psi}_{1}$ (resp. $\underline{\Psi}_{2}$ ) is any element in $\widehat{\boldsymbol{\Psi}}_{1}$ (resp. $\widehat{\Psi}_{2}$ ). Note that, thanks to (38), the generalized strains $\underset{\sim}{e}=\underline{\Psi} \cdot \nabla$ and $\underline{\Phi}=\underline{\Psi}^{d}$ associated to any $\underline{\boldsymbol{\Psi}}$ in $\widehat{\boldsymbol{\Psi}} \in \widehat{K C}$ are uniquely determined by $\widehat{\Psi}$ : the above right-hand side is hence well-defined. As above, we have

$$
\begin{aligned}
& \left|\widehat{a}\left(\widehat{\mathbf{\Psi}}_{1}, \widehat{\mathbf{\Psi}}_{2}\right)\right|=\left|a\left(\underline{\Psi}_{1}-\Pi\left(\underline{\Psi}_{1}\right), \underline{\Psi}_{2}-\Pi\left(\underline{\Psi}_{2}\right)\right)\right| \\
& \leq C\left\|\underline{\Psi}_{1}-\Pi\left(\underline{\Psi}_{1}\right)\right\|_{K C}\left\|\underline{\Psi}_{2}-\Pi\left(\underline{\Psi}_{2}\right)\right\|_{K C}=C\left\|\widehat{\Psi}_{1}\right\|_{\widehat{K C}}\left\|\widehat{\Psi}_{2}\right\|_{\widehat{K C}},
\end{aligned}
$$

which shows that $\widehat{a}$ is continuous on $\widehat{K C}$.

We consider the minimization problem

$$
\inf \{\widehat{\mathcal{P}}(\widehat{\underline{\Psi}}), \quad \widehat{\underline{\Psi}} \in \widehat{K C}\},
$$

where

$$
\widehat{\mathcal{P}}(\widehat{\underline{\mathbf{\Psi}}})=\frac{1}{2} \widehat{a}(\widehat{\underline{\mathbf{\Psi}}}, \widehat{\underline{\mathbf{\Psi}}})-\widehat{b}(\widehat{\underline{\mathbf{\Psi}}}) .
$$

To show that this problem is well-posed, we are left with showing the coercivity of $\widehat{a}$.

\subsection{Coercivity of the bilinear form $\widehat{a}$}

We have the following result:

Lemma 9. Let $\widehat{\Psi} \in \widehat{K C}$. There exists $c>0$ such that

$$
\|\underline{\Psi}\|_{K C^{d}} \leq\|\widehat{\Psi}\|_{\widehat{K C}} \leq c\|\Psi\|_{K C^{d}}
$$

where $\underline{\Psi}$ is any element in $\widehat{\underline{\Psi}}$ and the norm $\|\cdot\|_{K C^{d}}$ is defined by (37).

Recall that all the elements $\underline{\Psi}$ of $\widehat{\Psi} \in \widehat{K C}$ have the same deviatoric part, and that their spherical parts differ by an element of $\mathcal{R}$. The quantity $\|\underline{\Psi}\|_{K C^{d}}$ is hence the same for all $\underline{\Psi} \in \widehat{\underline{\Psi}}$.

Proof. Let $\widehat{\mathbf{\Psi}} \in \widehat{K C}$ and $\underline{\Psi} \in \widehat{\Psi}$. We have

$$
\|\widehat{\underline{\mathbf{\Psi}}}\|_{\widehat{K C}}^{2}=\|\underline{\mathbf{\Psi}}-\Pi(\underline{\mathbf{\Psi}})\|_{K C}^{2}=\|\underline{\boldsymbol{\Psi}}-\Pi(\underline{\mathbf{\Psi}})\|_{K C^{d}}^{2}+\left\|(\underline{\boldsymbol{\Psi}}-\Pi(\underline{\mathbf{\Psi}}))^{s}\right\|_{\boldsymbol{\sim}_{S}^{2}(\Omega)}^{2}
$$

hence

$$
\|\widehat{\underline{\Psi}}\|_{\widehat{K C}}^{2} \geq\|\underline{\Psi}-\Pi(\underline{\Psi})\|_{K C^{d}}^{2}=\|\underline{\Psi}\|_{K C^{d}}^{2} .
$$


Conversely, let $\underline{\boldsymbol{u}}$ be the vector associated to the spherical tensor $\underline{\boldsymbol{\Psi}}^{s}$ using (22). We have

$$
\left\|(\underline{\boldsymbol{\Psi}}-\Pi(\underline{\boldsymbol{\Psi}}))^{s}\right\|_{\boldsymbol{L}_{S}^{2}(\Omega)}^{2}=2\|\underline{\boldsymbol{u}}-\pi(\underline{\boldsymbol{u}})\|_{\underline{\boldsymbol{L}}^{2}(\Omega)}^{2}=2 \inf _{\underline{\boldsymbol{r}} \in \underline{\mathcal{R}}}\|\underline{\boldsymbol{u}}-\underline{\boldsymbol{r}}\|_{\underline{\boldsymbol{L}}^{2}(\Omega)}^{2}
$$

where $\pi$ is the orthogonal projection of $\underline{\boldsymbol{L}}^{2}(\Omega)$ on $\underline{\boldsymbol{\mathcal { R }}}$. Using (7) and (38), we deduce that

$$
\begin{aligned}
& \left\|(\underline{\boldsymbol{\Psi}}-\Pi(\underline{\boldsymbol{\Psi}}))^{s}\right\|_{\boldsymbol{\sim}_{S}^{2}(\Omega)}^{2} \leq C\|\underline{\boldsymbol{u}} \stackrel{s}{\otimes} \underline{\boldsymbol{\nabla}}\|_{{\underset{\sim}{H}}_{S}^{-1}(\Omega)}^{2} \leq 2 C\|\underline{\boldsymbol{\Psi}} \cdot \underline{\boldsymbol{\nabla}}\|_{\sim_{S}^{\boldsymbol{H}_{S}^{-1}(\Omega)}}^{2}+2 C\left\|\underline{\Psi}^{d} \cdot \underline{\boldsymbol{\nabla}}\right\|_{\sim}^{\boldsymbol{H}_{S}^{-1}(\Omega)} \\
& \leq 2 C\|\underline{\Psi} \cdot \underline{\boldsymbol{\nabla}}\|_{\sim}^{\boldsymbol{L}_{S}^{2}(\Omega)}+2 C\left\|\underline{\Psi}^{d}\right\|_{\underline{\boldsymbol{L}}_{S}^{2}(\Omega)}^{2}=2 C\|\underline{\Psi}\|_{K C^{d}}^{2} \text {. }
\end{aligned}
$$

We thus deduce from (49) that

$$
\|\widehat{\underline{\Psi}}\|_{\widehat{K C}}^{2} \leq\|\underline{\Psi}\|_{K C^{d}}^{2}+2 C\|\Psi\|_{K C^{d}}^{2} .
$$

Collecting this bound with (50), we conclude the proof of Lemma 9.

\subsection{Well-posedness of (48)}

We have shown above that $\widehat{b}$ is a continuous linear form on the Hilbert space $\widehat{K C}$, and that $\widehat{a}$ is a continuous bilinear symmetric coercive form. Using the Lax-Milgram theorem, we deduce the following result:

Theorem 10. Consider some external forces $\boldsymbol{f} \in \underline{L}^{2}(\Omega)$ that satisfy (45). Assume that the stiffness tensors $\underset{\approx}{C}$ and ${\underset{\Im}{\Phi}}^{\Phi}$ are symmetric and positive definite in the sense of (27). Then the minimization problem (48) admits a unique solution $\widehat{\Psi}^{\#}$.

Moreover, $\widehat{\boldsymbol{\Psi}}^{\#}$ is also the unique solution of the following problem: find $\widehat{\boldsymbol{\Psi}}^{\#}$ in $\widehat{K C}$ such that $\widehat{a}\left(\widehat{\underline{\Psi}}^{\#}, \widehat{\underline{\Psi}}\right)=\widehat{b}(\widehat{\underline{\Psi}})$ for all $\widehat{\underline{\Psi}}$ in $\widehat{K C}$.

We now build from $\widehat{\Psi}^{\#}$ a solution to the boundary value problem with free boundary conditions (16), (19), (24) and (44).

Theorem 11. Under the assumptions of Theorem 10, let $\widehat{\Psi}^{\#}$ be the unique solution to the minimization problem (48). Let $\underline{\Psi}^{\#}$ be any element in $\widehat{\mathbf{\Psi}}^{\#}$ and let

$$
\stackrel{e}{e}^{\#}=\underline{\Psi}^{\#} \cdot \underline{\nabla} \quad \text { and } \quad \underline{\Phi}^{\#}=\left(\underline{\Psi}^{\#}\right)^{d}
$$

be the generalized strain fields associated to $\underline{\Psi}^{\#}$ by the compatibility conditions (24). Recall these fields are independent of the choice of $\underline{\Psi}^{\#} \in \widehat{\underline{\Psi}}^{\#}$. Let

$$
\underline{\sim}^{\#}={\underset{\approx}{C}}^{e}: \stackrel{\sim}{\#}^{\#} \text { and } \quad \underline{\boldsymbol{R}}^{\#}={\underset{\Im}{\Phi}}^{\Phi} \therefore \underline{\Phi}^{\#}
$$

be the generalized stress fields associated to the generalized strain fields by the constitutive law (29).

Then ${\underset{\sim}{\sim}}^{\#}$ is in the space $S A(\underline{\boldsymbol{f}})$ defined by $(12)$ while $\underline{\boldsymbol{R}}^{\#}=\left(\stackrel{\boldsymbol{\sigma}}{\#}^{\#} \otimes \underline{\boldsymbol{\nabla}}\right)^{d}$. Moreover, $\boldsymbol{\sigma}^{\#}$ satisfies (44). 
Proof. We follow the same lines as in the proof of Theorem 7. According to the variational equation satisfied by $\widehat{\Psi}^{\#}$ (see Theorem 10), we have that, for all $\widehat{\Psi} \in \widehat{K C}$,

$$
\int_{\Omega} \boldsymbol{\sigma}^{\#}:(\underline{\boldsymbol{\Psi}} \cdot \underline{\boldsymbol{\nabla}})+\underline{\boldsymbol{R}}^{\#} \therefore \underline{\Psi}^{d}=\int_{\Omega} \frac{1}{2} \underline{f} \cdot(\underline{\Psi}: \underset{\sim}{\mathbf{1}}),
$$

where $\boldsymbol{\Psi}$ is any element in $\widehat{\underline{\Psi}}$. The above relation hence holds for any $\underline{\Psi} \in K C$. Following the arguments of the proof of Theorem 7, we obtain that $\boldsymbol{\sigma}_{\sim}^{\#} \in S A(\underline{\boldsymbol{f}})$ while $\underline{\boldsymbol{R}}^{\#}=$ $\left(\boldsymbol{\sigma}^{\#} \otimes \underline{\boldsymbol{\nabla}}\right)^{d}$.

It remains to show that $\boldsymbol{\sigma}^{\#}$ satisfies (44). Consider some $\underline{\Psi} \in \boldsymbol{C}_{S}^{\infty}(\Omega) \subset K C$. Integrating by part in (51), we deduce that

$$
\forall \underline{\Psi} \in C_{S}^{\infty}(\Omega), \quad \int_{\partial \Omega} \stackrel{\boldsymbol{\sigma}}{\sim}^{\#}:(\underline{\Psi} \cdot \underline{\boldsymbol{n}})=0 .
$$

We have used the fact that the trace of $\boldsymbol{\sigma}^{\#} \in \underset{\sim}{\boldsymbol{H}_{S}^{1}}(\Omega)$ is well defined on the regular boundary $\partial \Omega$. The quantity $\underline{\boldsymbol{\Psi}} \cdot \underline{\boldsymbol{n}}$ can be arbitrary prescribed on $\partial \Omega$, which implies that $\boldsymbol{\sigma}^{\#}=0$ on $\partial \Omega$.

\subsection{Link with a stress formulation}

In Theorem 11, we have built the generalized stress fields $\left(\boldsymbol{\sigma}_{\sim}^{\#}, \boldsymbol{R}^{\#}\right)$ solution to the boundary value problem with free boundary conditions. Furthermore, we have shown that $\underline{\boldsymbol{R}}^{\#}=\left(\boldsymbol{\sigma}^{\#} \otimes \underline{\boldsymbol{\nabla}}\right)^{d}$ and that $\boldsymbol{\sigma}_{\sim}^{\#} \in S A_{0}(\underline{\boldsymbol{f}})$, the set of statically admissible stress fields defined by

$$
S A_{0}(\underline{\boldsymbol{f}})=\{\underset{\boldsymbol{\sigma}}{\sim} \in S A(\underline{\boldsymbol{f}}), \quad \boldsymbol{\sigma} \text { satisfies }(44)\} .
$$

Similarly to the last assertion of Theorem 7 , we have the following result:

Theorem 12. Under the assumptions of Theorem 10 and with the notations of Theorem 11, the stress field $\boldsymbol{\sigma}^{\#}$ defined in Theorem 11 is the unique solution to the problem

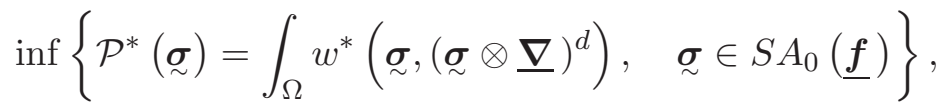

where $w^{*}$ is defined by (9).

Proof. The proof follows the same lines as the second part of that of Theorem 7, where $S A_{0}(\underline{\boldsymbol{f}})$ is substituted to $S A(\underline{\boldsymbol{f}})$. Let $\underset{\sim}{\boldsymbol{\sigma}}$ be any element in $S A_{0}(\underline{\boldsymbol{f}})$, and set $\boldsymbol{\sigma}^{\prime}=\boldsymbol{\sigma}-\boldsymbol{\sigma}_{\sim}^{\#}$. Then, by simple algebra, and using the fact that $\boldsymbol{\sigma}^{\prime} \cdot \underline{\boldsymbol{\nabla}}=0$, we have

$$
\mathcal{P}^{*}(\underset{\sim}{\boldsymbol{\sigma}})=\mathcal{P}^{*}\left(\underline{\sim}^{\#}\right)+\mathcal{P}^{*}\left(\boldsymbol{\sigma}^{\prime}\right)+\int_{\Omega} \underline{\sim}^{\prime}:\left(\underline{\Psi}^{\#} \cdot \underline{\boldsymbol{\nabla}}\right)+\left(\boldsymbol{\sigma}^{\prime} \otimes \underline{\boldsymbol{\nabla}}\right) \therefore \underline{\Psi}^{\#}
$$

By integration by part, and using that $\boldsymbol{\sigma}^{\prime}=0$ on $\partial \Omega$, we see that the last term in the above equation vanishes. We hence obtain that

$$
\mathcal{P}^{*}(\underset{\sim}{\boldsymbol{\sigma}})=\mathcal{P}^{*}\left({\underset{\sim}{\boldsymbol{\sigma}}}^{\#}\right)+\mathcal{P}^{*}\left({\underset{\sim}{\boldsymbol{\sigma}}}^{\prime}\right)
$$

and we eventually note that $\mathcal{P}^{*}\left(\boldsymbol{\sigma}^{\prime}\right)$ is positive as soon as $\boldsymbol{\sigma}^{\prime}$ does not vanish. Thus $\boldsymbol{\sigma}^{\#} \in S A_{0}(\underline{\boldsymbol{f}})$ is the unique solution to $(52)$. 


\section{Generalizations}

In this section, we point out various generalizations of the theory we have developed here.

\subsection{Body forces}

A straightforward generalization of the previous results is to consider body forces as fields $\underline{\boldsymbol{F}} \in \underline{\boldsymbol{L}}_{S}^{2}(\Omega)$, rather than fields $\underline{\boldsymbol{F}}^{s}$ constructed from $\underline{\boldsymbol{f}}$ following (42). We then replace the definition (36) by

$$
\forall \underline{\Psi} \in K C, \quad b(\underline{\Psi})=\int_{\Omega} \underline{\boldsymbol{F}} \therefore \underline{\Psi} .
$$

Consider the problem with clamped boundary conditions considered in Section 4. The linear form $b$ is again continuous on $K C_{0}$. The minimization problem (34) again has a unique solution $\underline{\Psi}^{\#}$, and Theorem 6 holds. The generalized stresses $\boldsymbol{\sigma}^{\#}$ and $\underline{\boldsymbol{R}}^{\#}$ defined from $\underline{\Psi}^{\#}$ as in Theorem 7 satisfy the following balance equations:

$$
\stackrel{\boldsymbol{\sigma}}{\sim}^{\#} \cdot \underline{\boldsymbol{\nabla}}+\underline{\boldsymbol{F}}: \underset{\sim}{\mathbf{1}}=0, \quad \underline{\boldsymbol{R}}^{\#}=\left(\stackrel{\boldsymbol{\sigma}}{*}^{\#} \otimes \underline{\boldsymbol{\nabla}}\right)^{d}+\underline{\boldsymbol{F}}^{d},
$$

which are substituted for (19). As a consequence, in the stress formulation associated to (34), one should be careful in the definition of the complementary energy $\mathcal{P}^{*}(\boldsymbol{\sigma})$. Likewise, the set of statically admissible stress fields should be defined as

$$
S A(\underline{\boldsymbol{F}})=\left\{\underset{\sim}{\boldsymbol{\sigma}} \in{\underset{\sim}{\boldsymbol{H}}}_{S}^{1}(\Omega), \quad \underset{\sim}{\boldsymbol{\sigma}} \cdot \underline{\boldsymbol{\nabla}}+\underset{\boldsymbol{F}}{\boldsymbol{\mathbf { 1 }}} \underset{\sim}{\mathbf{1}}=0 \text { on } \Omega\right\}
$$

instead of (12).

\subsection{More general strain energy densities}

Another generalization is to consider materials for which the strain energy density $w$ is a positive definite symmetric quadratic form of $(\underset{\sim}{e}, \Phi)$ that is not necessarily uncoupled in terms of $\underset{\sim}{e}$ and $\boldsymbol{\Phi}$. The density is then given by

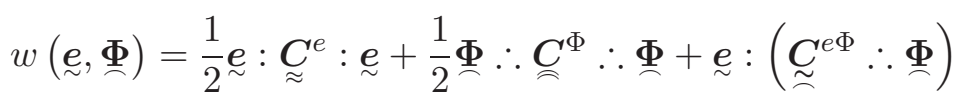

with the symmetries $C_{i j k l}^{e}=C_{j i k l}^{e}=C_{k l i j}^{e}, C_{i j k l m n}^{\Phi}=C_{j i k l m n}^{\Phi}=C_{l m n i j k}^{\Phi}$ and $C_{i j l m n}^{e \Phi}=$ $C_{j i l m n}^{e \Phi}=C_{i j m l n}^{e \Phi}$, and such that

$$
\forall(\underset{\sim}{e}, \underline{\Phi}) \in{\underset{\sim}{\mathbb{R}}}_{S} \times{\underset{\mathbb{R}}{S}}_{S}^{d}, \quad \beta(\underset{\sim}{e}: \underset{\sim}{e}+\underline{\Phi} \therefore \underline{\Phi}) \leq w(\underset{\sim}{\operatorname{s}}, \underline{\Phi})
$$

for some $\beta>0$. In this case, the generalized stresses $\boldsymbol{\sigma}$ and $\underline{\boldsymbol{R}}$, which are still defined by the constitutive equation (28), are given by

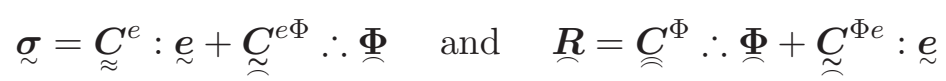

where the tensor ${\underset{\sim}{C}}^{\Phi e}$ is defined by $C_{l m n i j}^{\Phi e}=C_{i j l m n}^{e \Phi}$. The above constitutive law can be substituted for (29) in all the previous results. As pointed out in Remark 2, we can also consider heterogeneous materials. 


\subsection{Revisiting the free boundary conditions}

The free boundary conditions of the stress gradient theory impose that all the components of the second rank tensor $\boldsymbol{\sigma}$ vanish on the boundary $\partial \Omega$, see (44). However, the free boundary conditions in a standard Cauchy body impose that only the normal components $\underline{\boldsymbol{\sigma}} \cdot \underline{\boldsymbol{n}}$ vanish (recall that $\underline{\boldsymbol{n}}$ is the outer normal vector to $\partial \Omega$ ). If we want to prescribe only $\underline{\sigma} \cdot \underline{\boldsymbol{n}}=0$ in the stress gradient model, we must prescribe also the kinematic condition $\underline{\boldsymbol{\Psi}}^{d} \cdot \underline{\boldsymbol{n}}=0$ on the deviatoric part $\underline{\boldsymbol{\Psi}}^{d}$ of $\underline{\boldsymbol{\Psi}}$ in the following weak sense:

$$
\int_{\Omega} \underset{\sim}{\varphi}:\left(\underline{\Psi}^{d} \cdot \underline{\nabla}\right)+(\underset{\sim}{\varphi} \otimes \underline{\nabla}) \therefore \underline{\Psi}^{d}=0
$$

for all $\underset{\sim}{\varphi} \in \underset{\sim}{\boldsymbol{H}}{ }_{S}^{1}(\Omega)$.

We can generalize our results of the displacement formulation (namely Theorems 10 and 11$)$ to the case of the mixed boundary conditions $\underset{\sim}{\boldsymbol{\sigma}} \cdot \underline{\boldsymbol{n}}=0$ and $\underline{\Psi}^{d} \cdot \underline{\boldsymbol{n}}=0$ as follows:

- The space of kinematically compatible generalized displacements is chosen as the closed subspace $\widehat{K C}^{m} \subset \widehat{K C}$ such that the equation (53) holds true for all $\underset{\sim}{\varphi} \in$

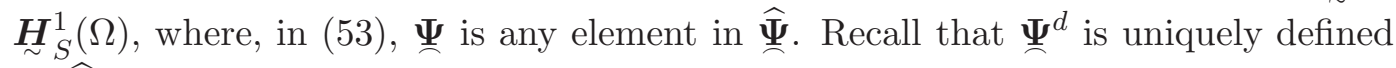
by $\widehat{\underline{\Psi}}$.

- The body forces $\underline{\boldsymbol{f}}$ are assumed to satisfy (45).

- Using the same arguments as for Theorem 10, we obtain that the minimization problem

$$
\inf \left\{\widehat{\mathcal{P}}(\widehat{\underline{\Psi}}), \quad \widehat{\underline{\Psi}} \in \widehat{K C}^{m}\right\}
$$

is well-posed.

- As in Theorem 11, we build the generalized stress fields ${\underset{\sim}{\boldsymbol{\sigma}}}^{\#}$ and $\underline{\boldsymbol{R}}^{\#}$, that satisfy (compare with (51))

$$
\forall \widehat{\mathbf{\Psi}} \in \widehat{K C}^{m}, \quad \forall \underline{\Psi} \in \widehat{\underline{\Psi}}, \quad \int_{\Omega} \underline{\sim}^{\#}:(\underline{\boldsymbol{\Psi}} \cdot \underline{\boldsymbol{\nabla}})+\underline{\boldsymbol{R}}^{\#} \therefore \underline{\mathbf{\Psi}}^{d}=\int_{\Omega} \frac{1}{2} \underline{\boldsymbol{f}} \cdot(\underline{\boldsymbol{\Psi}}: \underset{\sim}{\mathbf{1}}),
$$

where $\underline{\Psi}$ is any element in $\widehat{\underline{\Psi}}$. This implies that $\boldsymbol{\sigma}_{\sim}^{\#}$ is in the space $S A(\underline{\boldsymbol{f}})$ defined by (12) while $\underline{\boldsymbol{R}}^{\#}=\left(\stackrel{\boldsymbol{\sigma}}{*}^{\#} \otimes \underline{\boldsymbol{\nabla}}\right)^{d}$. We next deduce from (54) that

$$
\int_{\Omega} \underline{\boldsymbol{\sigma}}^{\#}:(\underline{\boldsymbol{\Psi}} \cdot \underline{\boldsymbol{\nabla}})+\left(\left(\boldsymbol{\sigma}^{\#} \otimes \underline{\boldsymbol{\nabla}}\right)^{d}\right) \therefore \underline{\Psi}^{d}=\int_{\Omega} \frac{1}{2} \underline{\boldsymbol{f}} \cdot(\underline{\mathbf{\Psi}}: \underset{\sim}{\mathbf{1}})
$$

hence

$$
\int_{\Omega} \underline{\boldsymbol{\sigma}}^{\#}:(\underline{\Psi} \cdot \underline{\boldsymbol{\nabla}})+\left(\boldsymbol{\sigma}^{\#} \otimes \underline{\boldsymbol{\nabla}}\right) \therefore \underline{\Psi}^{d}=\int_{\Omega} \frac{1}{2} \underline{\boldsymbol{f}} \cdot(\underline{\boldsymbol{\Psi}}: \underset{\sim}{\mathbf{1}}) .
$$

Using (53), we thus get

$$
\int_{\Omega} \boldsymbol{\sigma}^{\#}:\left(\underline{\Psi}^{s} \cdot \underline{\nabla}\right)=\int_{\Omega} \frac{1}{2} \underline{f} \cdot(\underline{\Psi}: \underset{\sim}{\mathbf{1}}) .
$$


Let $\underline{\boldsymbol{u}} \in \underline{\boldsymbol{H}}^{1}(\Omega)$, and consider the spherical third rank tensor $\underline{\Psi}^{s}$ associated to $\underline{\boldsymbol{u}}$ by (20). We consider the third rank tensor $\underline{\Psi}=\underline{\Psi}^{s}$, which is admissible in (54). Using (21), we see that (55) reads

$$
\int_{\Omega} \stackrel{\sigma}{\sim}^{\#}:(\underline{\boldsymbol{u}} \stackrel{s}{\otimes} \underline{\nabla})=\int_{\Omega} \underline{\boldsymbol{f}} \cdot \underline{\boldsymbol{u}} .
$$

Integrating by part and using the fact that $\underline{\boldsymbol{\sigma}}^{\#} \cdot \underline{\boldsymbol{\nabla}}+\underline{\boldsymbol{f}}=0$, we obtain that $\int_{\partial \Omega} \underline{\boldsymbol{u}}$. $\left(\underline{\boldsymbol{\sigma}}^{\#} \cdot \underline{\boldsymbol{n}}\right)=0$ for any $\underline{\boldsymbol{u}} \in \underline{\boldsymbol{H}}^{1}(\Omega)$. This implies that $\underline{\sim}^{\#} \cdot \underline{\boldsymbol{n}}=0$ on $\partial \Omega$.

Likewise, we can generalize Theorem 12 (stress formulation) to the case of the mixed boundary conditions $\boldsymbol{\sigma} \cdot \underline{\boldsymbol{n}}=0$ and $\underline{\Psi}^{d} \cdot \underline{\boldsymbol{n}}=0$. The set of statically admissible generalized stresses should be the closed subset $S A^{m}(\underline{\boldsymbol{f}}) \subset S A(\underline{\boldsymbol{f}})$ of stress fields such that $\boldsymbol{\sigma} \cdot \underline{\boldsymbol{n}}=0$ on $\partial \Omega$ (this condition is well-defined as $S \bar{A}(\underline{\boldsymbol{f}}) \subset \underset{\sim}{\boldsymbol{\boldsymbol { H } ^ { 1 }}}(\Omega)$ ).

\section{Conclusion}

Generalized energy principles have been established for the recent theory of stress gradient elasticity. They have been used to formulate existence and uniqueness theorems for the solutions of boundary value problems involving either clamping or stress free boundary conditions. The suitable clamping boundary conditions involve a combination of fixed normal components of the displacement vector and microdisplacement tensor, the latter being the additional kinematic degrees of freedom needed in the theory. The dual Neumann boundary conditions amount to fixing all components of the stress tensor on the boundary of the domain, which is a remarkable feature of the theory.

The stress gradient theory now stands as an alternative generalized continuum theory to the well-established strain gradient approach. Once the mathematical features of the theory have been settled, as done in the present work, it remains to address the physical questions raised by the model, such as the derivation of the higher order elastic moduli present in the model. This can be achieved for instance by means of generalized homogenization methods as tackled in the case of plate models by Lebée and Sab (2011a,b). Such homogenization schemes for architectured composite materials generally lead to anisotropic elastic properties.

The proposed stress gradient theory contains the usual fourth rank tensor of elastic moduli and a new sixth rank tensor of higher order moduli. The structure of the latter is similar to the sixth rank tensor arising in strain gradient elasticity. Note that, in the absence of central point symmetry, a fifth rank tensor coupling the stress and stress gradient tensors must be added to the constitutive model. This is a minor extension of the presented model. The theorems derived in the present work apply to this generalized case. The symmetry properties and corresponding symmetry group of these tensors can be studied following recent works by Olive and Auffray (2013); Auffray et al. (2013); Olive and Auffray (2014); Auffray (2014) for constitutive tensors of various orders.

The question of the performance of the stress gradient theory compared to the strain gradient one is raised for elasticity problems involving singularities, for instance at a crack 
tip, but also in the case of plasticity where stress gradient plasticity could emerge as an alternative to widely used strain gradient plasticity (Chakravarthy and Curtin, 2011). Finite element implementation of the stress gradient model is the next step in this direction to solve new boundary value problems in the mechanics of materials.

\section{References}

\section{References}

R.A. Adams. Sobolev spaces. Academic Press, San Diego, 1975.

C. Amrouche, P.G. Ciarlet, L. Gratie, and S. Kesavan. On the characterizations of matrix fields as linearized strain tensor fields. Journal de Mathématiques Pures et Appliquées, $86: 116-132,2006$.

N. Auffray. Analytical expressions for odd-order anisotropic tensor dimension. Comptes Rendus Mécanique, 342:284-291, 2014.

N. Auffray, H. Le Quang, and Q.C. He. Matrix representations for 3D strain-gradient elasticity. Journal of the Mechanics and Physics of Solids, 61:1202-1223, 2013.

J.L. Bleustein. A note on the boundary conditions of Toupin's strain-gradient theory. Int. J. Solids Structures, 3:1053-1057, 1967.

S.S. Chakravarthy and W.A. Curtin. Stress-gradient plasticity. Proceedings of the National Academy of Sciences of the United States of America, 108:15716-15720, 2011.

F. Dell'Isola and P. Seppecher. The relationship between edge contact forces, double forces and intersticial working allowed by the principle of virtual power. C.R. Acad. Sci. Paris IIb, 321:303-308, 1995.

F. Dell'Isola and P. Seppecher. Edge contact forces and quasi-balanced power. Meccanica, 32:33-52, 1997.

F. Dell'Isola, P. Seppecher, and A. Madeo. How contact interactions may depend on the shape of Cauchy cuts in N-th gradient continua: approach "à la D'Alembert". Zeitschrift für Angewandte Mathematik und Physik, 63:1119-1141, 2012.

A.C. Eringen. Nonlocal continuum field theories. Springer, New York, 2002.

S. Forest and E.C. Aifantis. Some links between recent gradient thermo-elasto-plasticity theories and the thermomechanics of generalized continua. Int. J. Solids Structures, 47: 3367-3376, 2010.

S. Forest and K. Sab. Stress gradient continuum theory. Mechanics Research Communications, 40:16-25, 2012.

P. Germain. La méthode des puissances virtuelles en mécanique des milieux continus, première partie : théorie du second gradient. J. de Mécanique, 12:235-274, 1973. 
D. Iesan. Thermoelasticity of bodies with microstructure and microtemperatures. Int. J. Solids Structures, 44:8648-8662, 2007.

D. Iesan and R. Quintanilla. Existence and continuous dependence results in the theory of microstretch elastic bodies. International Journal of Engineering Science, 32:991-1001, 1994.

D. Iesan and R. Quintanilla. On a strain gradient theory of thermoviscoelasticity. Mechanics Research Communications, 48:52-58, 2013.

J. Jeong and P. Neff. Existence, uniqueness and stability in linear Cosserat elasticity for weakest curvature conditions. Mathematics and Mechanics of Solids, 15:78-95, 2010.

A. Lebée and K. Sab. A bending-gradient model for thick plates, Part I: Theory. Int. J. Solids Structures, 48:2878-2888, 2011a.

A. Lebée and K. Sab. A bending-gradient model for thick plates, Part II: Closed-form solutions for cylindrical bending of laminates. Int. J. Solids Structures, 48:2889-2901, 2011b.

R.D. Mindlin. Second gradient of strain and surface-tension in linear elasticity. Int. J. Solids Structures, 1:417-438, 1965.

R.D. Mindlin and N.N. Eshel. On first strain gradient theories in linear elasticity. Int. J. Solids Structures, 4:109-124, 1968.

J.J. Moreau. Duality characterization of strain tensor distributions in an arbitrary open set. Journal of Mathematical Analysis and Applications, 72:760-770, 1979.

J. Necas. Les méthodes directes en théorie des équations elliptiques. Masson, Paris, 1967.

P. Neff and S. Forest. A geometrically exact micromorphic model for elastic metallic foams accounting for affine microstructure. Modelling, existence of minimizers, identification of moduli and computational results. Journal of Elasticity, 87:239-276, 2007.

M. Olive and N. Auffray. Symmetry classes for even-order tensors. Mathematics and Mechanics of Complex Systems, 1:177-210, 2013.

M. Olive and N. Auffray. Symmetry classes for odd-order tensors. ZAMM-Zeitschrift für Angewandte Mathematik und Mechanik, 94:421-447, 2014.

C. Polizzotto. Stress gradient versus strain gradient constitutive models within elasticity. Int. J. Solids Structures, 51:1809-1818, 2014.

C. Polizzotto. A unifying variational framework for stress gradient and strain gradient elasticity theories. European Journal of Mechanics A-Solids, 49:430-440, 2015.

C.Q. Ru and E.C. Aifantis. A simple approach to solve boundary value problems in gradient elasticity. Acta Mechanica, 101:59-68, 1993. 
R.A. Toupin. Elastic materials with couple stresses. Arch. Rat. Mech. Anal., 11:385-414, 1962. 